\title{
Article
}

\section{In Silico Studies of Tumor Targeted Peptide-Conjugated Natural Products for Targeting Over-Expressed Receptors in Breast Cancer Cells Using Molecular Docking, Molecular Dynamics and MMGBSA Calculations}

\author{
Lucy R. Hart, Charlotta G. Lebedenko, Saige M. Mitchell, Rachel E. Daso and Ipsita A. Banerjee *
}

Citation: Hart, L.R.; Lebedenko, C.G.; Mitchell, S.M.; Daso, R.E.; Banerjee,

I.A. In Silico Studies of Tumor

Targeted Peptide-Conjugated Natural Products for Targeting

Over-Expressed Receptors in Breast Cancer Cells Using Molecular Docking, Molecular Dynamics and MMGBSA Calculations. Appl. Sci. 2022, 12, 515. https://doi.org/ 10.3390/app12010515

Academic Editor: Lidia Feliu

Received: 25 November 2021 Accepted: 28 December 2021 Published: 5 January 2022

Publisher's Note: MDPI stays neutral with regard to jurisdictional claims in published maps and institutional affiliations.

Copyright: (c) 2022 by the authors. Licensee MDPI, Basel, Switzerland. This article is an open access article distributed under the terms and conditions of the Creative Commons Attribution (CC BY) license (https:// creativecommons.org/licenses/by/ $4.0 /)$.

\author{
Department of Chemistry, Fordham University, 441 East Fordham Road, Bronx, NY 10458, USA; \\ lhart4@fordham.edu (L.R.H.); clebedenko@fordham.edu (C.G.L.); smitchell27@fordham.edu (S.M.M.); \\ rdaso@fordham.edu (R.E.D.) \\ * Correspondence: banerjee@fordham.edu
}

\begin{abstract}
In this work, in silico studies were carried out for the design of diterpene and polyphenolpeptide conjugates to potentially target over-expressed breast tumor cell receptors. Four point mutations were induced into the known tumor-targeting peptide sequence YHWYGYTPQN at positions 1, 2, 8 and 10, resulting in four mutated peptides. Each peptide was separately conjugated with either chlorogenate, carnosate, gallate, or rosmarinate given their known anti-tumor activities, creating dual targeting compounds. Molecular docking studies were conducted with the epidermal growth factor receptor (EGFR), to which the original peptide sequence is known to bind, as well as the estrogen receptor $(\mathrm{ER} \alpha)$ and peroxisome proliferator-activated receptor (PPAR $\alpha)$ using both Autodock Vina and FireDock. Based on docking results, peptide conjugates and peptides were selected and subjected to molecular dynamics simulations. MMGBSA calculations were used to further probe the binding energies. ADME studies revealed that the compounds were not CYP substrates, though most were Pgp substrates. Additionally, most of the peptides and conjugates showed MDCK permeability. Our results indicated that several of the peptide conjugates enhanced binding interactions with the receptors and resulted in stable receptor-ligand complexes; Furthermore, they may successfully target ER $\alpha$ and PPAR $\alpha$ in addition to EGFR and may be further explored for synthesis and biological studies for therapeutic applications.
\end{abstract}

Keywords: peptide conjugates; polyphenols; diterpenes; molecular dynamics; docking

\section{Introduction}

Peptide-based cancer therapies are gaining importance, as they exhibit high biocompatibility compared to traditional chemotherapeutics and can also be used to specifically target cancer cells [1]. Tumor cell targeting can be accomplished by developing specific peptide sequences for several components typically overexpressed in cancer cells, such as receptors, integrins on tumor blood vessels, extracellular matrix components, and tumor-associated macrophages [2,3]. Molecular modeling and computational methods are being increasingly used to examine binding interactions and screen small molecules and peptides for developing more efficient and targeted compounds [4]. In one study, short peptides composed of Phe and Asp residues were used to target overexpressed PTP1B and SHP2 phosphatases in breast cancer cells; the peptides were shown by molecular modeling techniques to bind to the phosphatases and demonstrated inhibition against MCF-7 breast tumor cells [5]. In another study, computational methods were used to predict tumor-targeting ability of peptides for the epidermal growth factor receptor (EGFR) [6]. Peptide vaccines are an additional type of peptide-based cancer therapy, used to activate tumor-associated antigenspecific immune responses through processes such as targeting major histocompatibility complexes (MHCs) in tumor-associated antigen-presenting cells [7]. 
In numerous studies, targeted peptides have been conjugated with drugs to enhance toxicity toward cancer cells and to alleviate adverse side effects caused by lack of specificity in chemotherapeutic drugs [8,9]. For example, the peptide-drug conjugate ${ }^{177}$ Lu-dotatate, composed of somatostatin conjugated with the cytotoxic radiotherapeutic agent ${ }^{177} \mathrm{Lu}$, was used to treat gastroenteropancreatic neuroendocrine tumors [10]. Recently, the peptide angiopep-2 was conjugated with the drug paclitaxel, resulting in an increased blood-brain barrier permeation without increasing toxicity [11]. In another study, $\mathrm{pH}$ low insertion peptide (pHLIP), a 36-amino acid peptide derived from bacteriorhodopsin, was conjugated with doxorubicin; it demonstrated cancer cell-specific cytotoxicity in MCF-7 breast tumor cells [12]. In a separate study, chlorambucil and camptothecin-peptide conjugates were found to bind to integrin in conformations similar to the bio-active conformation of the parent peptide, as exhibited by molecular dynamics simulations, indicating their potential for use as targeting agents [13].

Naturally occurring plant polyphenols and diterpene derivatives are known for their antioxidant and anticancer properties. These phytochemicals are nontoxic, many are found in the human diet and are therefore promising tools in cancer research $[14,15]$. Various mechanisms have been suggested for the anticancer activity of polyphenols [16]. For example, carnosic acid and rosmarinic acid, both derived from the rosemary plant (Rosmarinus officinalis L.), have been found to exhibit antioxidant properties and anti-carcinogenesis in both tumor initiation and promotion phases [17]. Chlorogenic acid is a component of coffee and many other plants and has been found to possess antioxidant, antibacterial, and anticarcinogenic properties [18]. Gallic acid and curcumin, derived from plant or root sources, have shown antiproliferative properties and can induce apoptosis in cancer cells through regulation of Bax, Bcl-2, and X-linked inhibitor of apoptosis protein (Xiap) $[19,20]$. Using computational methods, it was recently reported that the polyphenol quercetin binds to RSK2, a ribosomal kinase which is involved in prostate cancer proliferation [21]. In another study, computational docking and screening methods were used to identify the targeting ability of the green tea derived polyphenol epigallocatechin gallate (EGCG) toward RSK2 as a novel molecular target [22,23].

Both molecular docking and molecular dynamics (MD) studies are useful in determining the efficacy and stability of receptor targeting with various ligands [24]. For example, in a recent study, it was shown that polyphenols such as papyriflavonol A and kazinol $\mathrm{F}$ interacted with the main protease (MPro) enzyme of SARS-CoV-2 and may be developed as potential drugs [25]. In another study, Jeong and coworkers tested several polyphenols using docking and MD and showed that the methoxy flavonoid acacetin formed a stable complex with the active site of aldose reductase receptor; thus, it may potentially be developed as an inhibitor of the enzyme [26].

In this work, we have designed anticancer peptides that were conjugated with chlorogenic acid (CGA), carnosic acid (CSA), gallic acid (GLA), and rosmarinic acid (RMA) to develop new polyphenol-peptide conjugates for targeting receptors over-expressed in breast cancer cells. We chose to study three receptors. Estrogen receptor alpha $(\mathrm{ER} \alpha)$, which is over-expressed in hormone sensitive breast cancer; peroxisome proliferator-activated receptor alpha (PPAR- $\alpha)$, which is over-expressed in triple negative breast (TNBC) and ER positive cancer cells [27]; and epidermal growth factor receptor (EGFR), which is also over-expressed in TNBC [28]. The anticancer peptides were designed by performing point mutations on the known antitumor peptide sequence Y-H-W-Y-G-Y-T-P-Q-N (Pep). This peptide sequence is a truncated form of Y-H-W-Y-G-Y-T-P-Q-N-V-I; both sequences have previously been shown to bind to epidermal growth factor receptor (EGFR) [29]. Additionally, in recent studies, it has been shown that there is involvement of crosstalk between estrogen receptors (ERs) and EGFR in breast cancer cells and that expression of extracellular secreted and cell bound proteoglycans are involved in cancer progression [30]. We therefore sought to probe the possible targeting abilities of this sequence not only for EGFR, but also for ER- $\alpha$, as well as alternative receptors overexpressed in breast cancer cells (PPAR- $\alpha)$. To enhance receptor targeting, we designed four peptide sequences by 
point mutation of the original peptide Y-H-W-Y-G-Y-T-P-Q-N at positions 1, 2, 8, and 10. For Mutation 1, Y, which was in the first position, was substituted with $\mathbf{F}$, thus designing the sequence F-H-W-Y-G-Y-T-P-Q-N (Mut1). For Mutation 2, H, which was in the second position, was substituted with I, producing the sequence Y-I-W-Y-G-Y-T-P-Q-N (Mut2) and significantly altering the properties. For Mutation 3, $\mathbf{P}$ at position 8 was changed to $\mathbf{H}$, making Y-H-W-Y-G-Y-T-H-Q-N (Mut3). For Mutation 4, N at position 10 was substituted with D, making Y-H-W-Y-G-Y-T-P-Q-D (Mut4). Thus, Mut1 replaces Y with F, which are both hydrophobic. In previous studies, as indicated by Ariani and co-workers [29] replacing $\mathrm{Y}$ to $\mathrm{F}$ increased cell-adhesion toward both MCF-7 and MDA-MB-231 cells. Mutation 2 was intended to explore the effect of increased hydrophobicity by changing H to I. Mutation 3, which substituted Pro with His, was intended to increase the polarity of the peptide and reduce the effects of rigidity induced by Pro. Finally, $\mathrm{N}$ was substituted by D to examine the effect of a negatively charged residue at the C-terminal. Each of these peptides were then conjugated to CGA, CSA, GLA or RMA. The sequences of the peptides and chemical structures of the conjugates are shown in Tables 1 and 2. The binding affinities of each of the peptides and their conjugates was compared on the basis of molecular docking abilities with ER- $\alpha$ (PDB ID: 3OS8), Epidermal Growth Factor Receptor (EGFR) (PDB ID: 2RGP), and peroxisome proliferator-activated receptor alpha (PPAR- $\alpha$ ) (PDB ID: 2P54) in order to evaluate potential targeting abilities of these conjugates. We further conducted molecular docking studies with the unmutated original peptide (pep) sequence as a point of comparison. In several cases, the novel conjugates displayed comparable or improved binding affinities for ER compared to ER's native ligand estradiol [31]. The stability and compactness of the receptor-docked ligands were examined using MD simulations and average binding energies were estimated using MMGBSA calculations from the trajectories.

Table 1. Peptide Sequences.

\begin{tabular}{cc}
\hline Original Peptide (Pep) & YHWYGYTPQN \\
\hline Mutation 1 (Mut) & FHWYGYTPQN \\
\hline Mutation 2 (Mut2) & YIWYGYTPQN \\
\hline Mutation 3 (Mut3) & YHWYGYTHQN \\
\hline Mutation 4 (Mut4) & YHWYGYTPQD \\
\hline
\end{tabular}

Overall, in this study, we were able to refine the targeting ability of the peptide $Y$ H-W-Y-G-Y-T-P-Q-N by inducing point mutations, conjugating it to polyphenols, and assessing targeting of ER- $\alpha$, PPAR- $\alpha$ and EGFR. We found that several peptide conjugates possessed comparable targeting abilities for all three receptors, while many of the mutated peptides and conjugates exhibited improved binding. Thus, we have determined for the first time that the tumor targeting peptide, its single-point mutated peptides, and some of the novel diterpene/polyphenol-peptide conjugates may be beneficial for developing drugs for targeting of ER- $\alpha$ and EGFR. The capability of these peptides and peptide conjugates to bind to PPAR- $\alpha$ also indicates their potential for targeting several other types of cancer cells. Overall, the conjugates that exhibited the most promising binding interactions for all three receptors were Rosmarinate-Y-H-W-Y-G-Y-T-P-Q-N, Gallate-F-H-W-Y-G-Y-T-P-Q-N and Gallate-Y-I-W-Y-G-Y-T-P-Q-N. 
Table 2. Structures of Polyphenol-peptide Conjugates with Pep and Mut1-Mut4. Sequences from top to bottom. (Column A = carnosic amide derived conjugates; Column $\mathrm{B}=$ Chlorogenic amide conjugates; Column $\mathrm{C}=$ gallic amide onjugates and Column $\mathrm{D}=$ Rosmarinic amide derived conjugates).

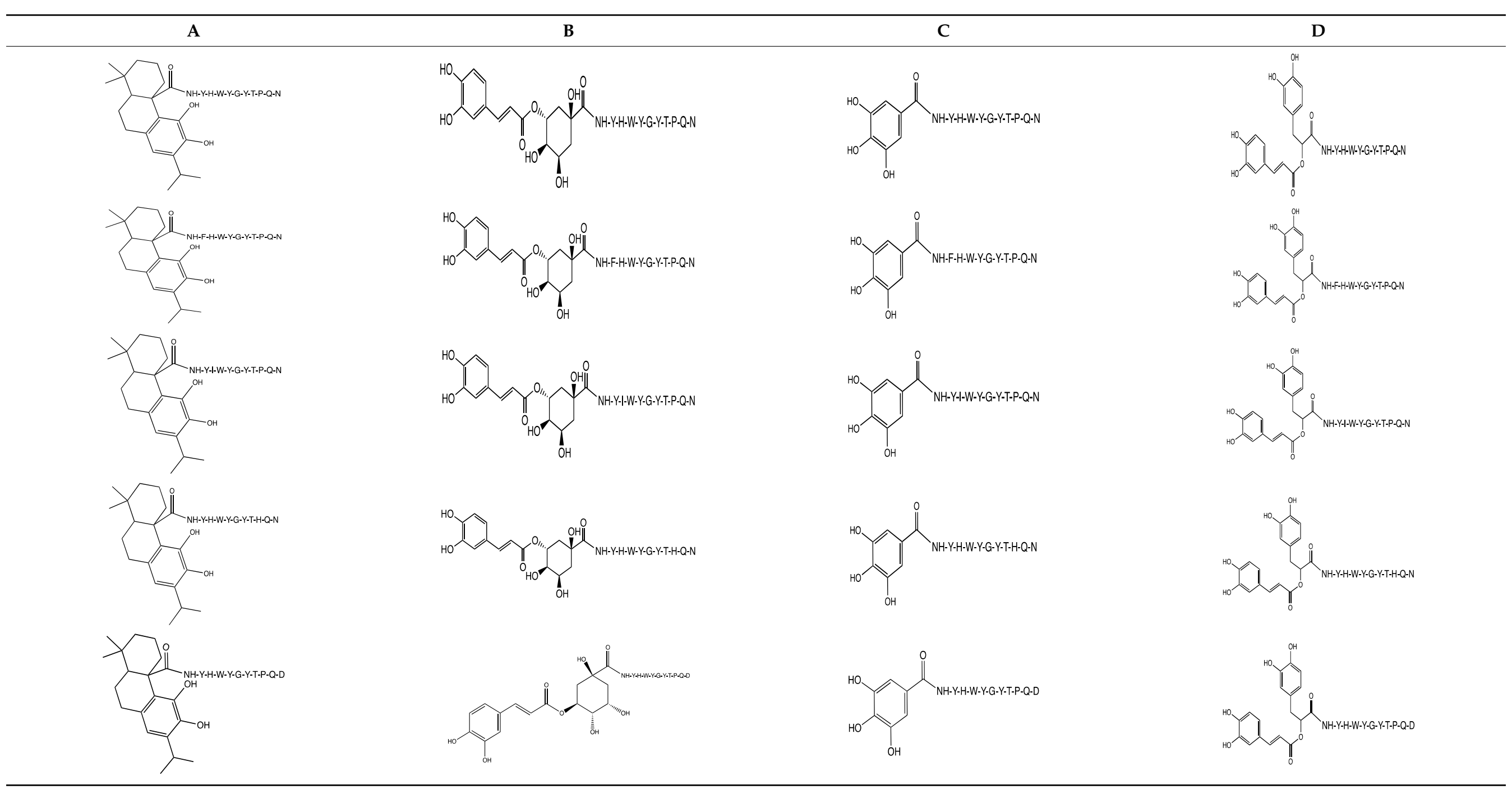




\section{Methods}

\subsection{Peptide and Conjugate Design}

Peptides were selected on the basis of their anticancer activity as determined by the AntiCP database [32,33]. The peptide Y-H-W-Y-G-Y-T-P-Q-N (Pep), was utilized as the original peptide to which four single point mutations were carried out in order to design the following peptides: F-H-W-Y-G-Y-T-P-Q-N (Mut1), Y-I-W-Y-G-Y-T-P-Q-N (Mut2), Y-HW-Y-G-Y-T-H-Q-N (Mut3), and Y-H-W-Y-G-Y-T-P-Q-D (Mut4); containing point mutations at positions 1, 2, 8, and 10, respectively. The diterpene carnosic acid (CSA) or polyphenols chlorogenic acid (CGA), gallic acid (GLA), and rosmarinic acid (RMA) were selected on the basis of their anticancer and antioxidant activity. Peptides and peptide conjugates were drawn in ChemDraw 18.1, then energy minimized and converted to PDB files in Chem3D. Molecules were visualized in PyMOL v. 2.4.0 [34]. To conjugate the peptides, the free carboxyl groups of the CGA, CSA, GLA or RMA were conjugated with the free N-terminal amino group of each of the peptides.

\subsection{I-TASSER Studies}

I-TASSER studies were conducted for each peptide using the I-TASSER web server in order to predict secondary structures and solvent accessibility [35-38].

\subsection{Binding Pocket Analysis}

Binding pocket analysis was performed on each receptor using the Pocket-Cavity Search Application (POCASA) web server, which probes the surface of proteins of known structure to identify potential ligand binding pockets and cavities [39]. X-ray crystal structures of the receptors were retrieved from the RCSB Protein Data Bank. Before uploading each of the files on the web server, any attached ligands were first removed using PyMOL and the receptors were saved as PDB files. For ER, PDB ID: 3 OS8 [40]; for PPAR- $\alpha$, PDB ID: 2P54 [41]; and for EGFR, PDB ID: 2RGP [42] were used.

\subsection{Receptor-Ligand Docking Studies}

Molecular docking studies were conducted using two different software because it has been shown that comparing multiple docking software aids in validation of the results. Software used were Autodock Vina v. 1.1.2 (MacOSX 64-bit, beta version) [43] and FireDock, in combination with PatchDock [44,45]. For Autodock Vina, receptor and ligand files were prepared and converted into PDBQT format using Autodock Tools. System-determined docking grids (dimensions $40 \AA \times 40 \AA \times 40 \AA$ ) were used for each receptor. Each peptide and polyphenol-peptide conjugate was run through Autodock Vina with each receptor to determine maximal binding affinity and ideal docking configurations. Additional molecular docking studies were conducted with each peptide and polyphenol-peptide conjugate with each receptor using the PatchDock web server. PatchDock conducts molecular docking using shape complementarity principles. PDB files for each receptor and ligand were uploaded to the PatchDock web server and submitted for docking using a cluster RMSD value of 4.0. Molecular docking data obtained through PatchDock was further analyzed using the Fast Interaction Refinement in Molecular Docking (FireDock) online server. PDB files for each receptor and ligand were uploaded to the FireDock server, along with transformations files generated for the corresponding complexes through PatchDock. From FireDock, global energy values were obtained for each receptor-ligand complex.

\subsection{Protein-Ligand Interaction Determination}

Receptor-ligand configurations were then run through the Protein-Ligand Interaction Profiler (PLIP) online interface to determine the major interactions contributing to docking ability for each combination [46]. 


\subsection{Molecular Dynamics Studies}

Receptor-ligand molecular dynamics studies were conducted using Desmond in Schrödinger Suites version 2020-3 [47] for $100 \mathrm{~ns}$ with each of the peptide-receptor or polyphenol-peptide conjugate-receptor complexes. Receptors and ligands were prepared in PyMOL and Maestro. Using Protein Preparation Wizard, hydrogens and any missing side chains were added to the receptors and disulfide bonds were created. $\mathrm{H}$-bonds were optimized using a $\mathrm{pH}$ of 7.0 and restrained minimization was conducted using the OPLS3e force field, while heavy atoms were converged to $0.30 \AA$ RMSD. Simulations were set up in a $10 \AA \times 10 \AA \times 10 \AA$ orthorhombic box with the SCP solvent model and OPLS3e force field. Systems were neutralized with sodium or chloride ions. Protein-ligand RMSD, RMSF, and simulation interaction interpretations were generated by Desmond. Desmond was run on a Linux operating system: Linux (Ubuntu 18.0.4 LTS) with 24 CPUs, and 4 GPUs, Intel(R) Xeon(R) CPU E5-2690 v3 @ 2.60GHz.

\subsection{MMGBSA Energy Calculations}

To evaluate the theoretical free energies of binding of the peptides and the conjugates with the receptors, we have utilized the molecular mechanics generalized Born surface area (MM-GBSA) method to calculate the relative binding free energies [48]. The free energy of binding can be calculated as $\Delta \mathrm{G}$ (bind) $=\Delta \mathrm{G}$ (solv) $+\Delta \mathrm{E}(\mathrm{MM})+\Delta \mathrm{G}(\mathrm{SA})$ where $\Delta \mathrm{G}$ solv is the difference in solvation energy of the ligand-bound receptor complex and the sum of the solvation energies for the free ligand and receptor [49]. $\Delta \mathrm{E}(\mathrm{MM})$ is the difference in the minimized energies between receptor-ligand complex and the sum of the energies of each of the free ligands and receptor while $\Delta \mathrm{G}(\mathrm{SA})$ is the difference in surface area energies of the ligand-receptor complex and the sum of the surface area energies for each of the neat ligands and receptor. The polar effect of free energy is examined by a generalized Born model with an external dielectric constant of 80 and an internal dielectric constant of 1 , while the non-polar energy contribution is calculated from the solvent accessible surface area (SASA). The Prime module of the Schrodinger Suite 2020-03 was used to calculate the free energies [50]. Output trajectory files from each MD simulation were analyzed using the script thermal_mmgbsa.py. The average free energy of each receptor-ligand complex through the $100 \mathrm{~ns}$ MD simulation was determined.

\subsection{ADME Studies}

Absorption, distribution, metabolism, and excretion (ADME) data were obtained for each peptide and selected polyphenol-peptide conjugates using the ADMETlab2.0 webserver [51]. These studies were used to predict the partition coefficient, MDCK cell permeability, CYP and Pgp substrate/inhibitor properties, of each peptide and conjugate.

\section{Results and Discussion}

To determine the binding affinity of the designed polyphenol-peptide conjugates as well as the peptides, we examined three different receptors. The binding affinity of the four new sequences with single point mutations and the original peptide (Pep) toward ER- $\alpha$, PPAR- $\alpha$, and EGFR was explored. We specifically chose those four positions and amino acids to mutate in order to examine the effect of H-bonding, hydrophobicity or charge alteration on the binding affinity. To further enhance tumor targeting and increase potency, each of the peptides were conjugated with the polyphenols CGA, CSA, GLA, and RMA, each of which are well-known for their inherent anticancer properties.

\subsection{Anti CP Studies}

The peptide and each of the mutated variants were found to possess anticancer properties according to AntiCP analysis (Table 3). Mut1 had the highest SVM score and was predicted to possess anticancer properties with the greatest degree, followed by Pep, Mut4, Mut3, and Mut2. The pI values indicated that Mut2 and Mut4 are slightly acidic in an aqueous environment, while Pep, Mut1, and Mut 4 are relatively neutral. As 
expected, mutating YHWYGYTPQN to YIWYGYTPQN (Mutation 3) showed the highest change in hydrophobicity, where it increased from -0.17 to -0.03 , while Mutation 4 (YHWYGYTPQD) showed the highest hydrophilicity at -0.80 .

Table 3. Properties of Designed Anticancer Peptides, (a) Peptide AntiCP Results, (b) I-TASSER Results.

\begin{tabular}{|c|c|c|c|c|c|c|}
\hline Peptide Sequence & Mutation Position & SVM Score & Hydrophobicity & Hydropathicity & Hydrophilicity & pI \\
\hline YHWYGYTPQN & None & 0.88 & -0.14 & -1.77 & -1.08 & 7.08 \\
\hline FHWYGYTPQN & 1 & 1.00 & -0.08 & -1.36 & -1.10 & 7.09 \\
\hline YIWYGYTPQN & 2 & 0.77 & -0.03 & -1.00 & -1.21 & 5.87 \\
\hline YHWYGYTHQN & 8 & 0.82 & -0.17 & -1.93 & -1.13 & 7.25 \\
\hline YHWYGYTPQD & 10 & 0.85 & -0.15 & -1.77 & -0.80 & 5.09 \\
\hline Peptide Sequence & \multicolumn{2}{|c|}{ Secondary Structure } & \multicolumn{2}{|c|}{$\begin{array}{c}\text { Solvent Accessibility/Residue } \\
\text { in the Sequence * }\end{array}$} & \multicolumn{2}{|c|}{ C Score } \\
\hline YHWYGYTPQN & \multicolumn{2}{|c|}{ СССССССССС } & \multicolumn{2}{|c|}{$7-4-3-4-4-3-4-4-7-8$} & \multicolumn{2}{|l|}{-0.58} \\
\hline FHWYGYTPQN & \multicolumn{2}{|c|}{ СССССССССС } & \multicolumn{2}{|c|}{$6-4-3-4-4-3-4-4-7-8$} & \multicolumn{2}{|l|}{-0.56} \\
\hline YIWYGYTPQN & \multicolumn{2}{|c|}{ CCSSCCCCCC } & \multicolumn{2}{|c|}{$6-3-2-4-4-3-4-4-7-8$} & \multicolumn{2}{|l|}{-0.54} \\
\hline YHWYGYTHQN & \multicolumn{2}{|c|}{ СССССССССС } & \multicolumn{2}{|c|}{$7-4-3-4-4-3-4-4-7-8$} & \multicolumn{2}{|l|}{-0.68} \\
\hline YHWYGYTPQD & \multicolumn{2}{|c|}{ СССССССССС } & \multicolumn{2}{|c|}{$7-4-3-4-4-3-4-4-7-8$} & \multicolumn{2}{|l|}{-0.46} \\
\hline
\end{tabular}

* Numbers given are for each residue in the sequence, in the range of 0 to 9 , with 9 having the highest. solvent exposure and 0 having lowest solvent exposure.

\subsection{I-TASSER Studies}

In order to predict the structures of the peptides, we carried out I-TASSER studies. As shown in 3, I-TASSER studies predicted exclusively coiled structures for all peptides except Mut2, for which Trp3 and Tyr4 regions were predicted to form strands based on C-scores. This secondary structural change is likely a result of the change of His to Ile, imparting elevated hydrophobicity to the peptide. Solvent accessibility ranged from 6-8 for N- and C-terminal residues and from 2-4 for interior residues. Solvent accessibility for the original peptide (Pep) was found to be 7-4-3-4-4-3-4-4-7-8 corresponding to each residue of the sequence. For Mut1, the N-terminal solvent accessibility decreased slightly, giving a value of 6-4-3-4-4-3-4-4-7-8. For Mut2, where His2 was changed to Ile, scores were found to be 6-3-2-4-4-3-4-4-7-8. Mut3 and Mut4 both had identical solvent accessibility to Pep, 7-4-3-4-4-3-4-4-7-8. These results indicate that the peptides had amphiphilic properties. The C-scores, or confidence scores, for I-TASSER predictions were -0.58 for Pep, -0.56 for Mut1, -0.54 for Mut2, -0.68 for Mut3, and -0.46 for Mut4. Thus, the I-TASSER predictions can be expected to have the most variability for Mut3 and the least for Mut4 based on a higher confidence level for these predictions.

\subsection{Receptor Binding Pocket Analysis}

Potential ligand binding pockets of ER- $\alpha$, PPAR- $\alpha$, and EGFR were predicted by the POCASA web server. Predicted binding pockets are shown in Figure 1. For ER $\alpha$, the pocket ranked number 1 by POCASA had a volume of $233 \AA$ and a VD of 2355. Pockets 2 and 3 had volumes of $87 \AA$ and $74 \AA$ and VD of 420 and 180, respectively. The large diversity of pocket volumes and VD for ER indicate that this receptor can bind to a variety of ligands; thus it was predicted to potentially interact favorably with the designed conjugates and peptides. Studies have shown that when several naturally occurring flavonoids were docked to ER, many interacted with Glu353, Arg394, Thr347, and Asp351 residues [52]. 

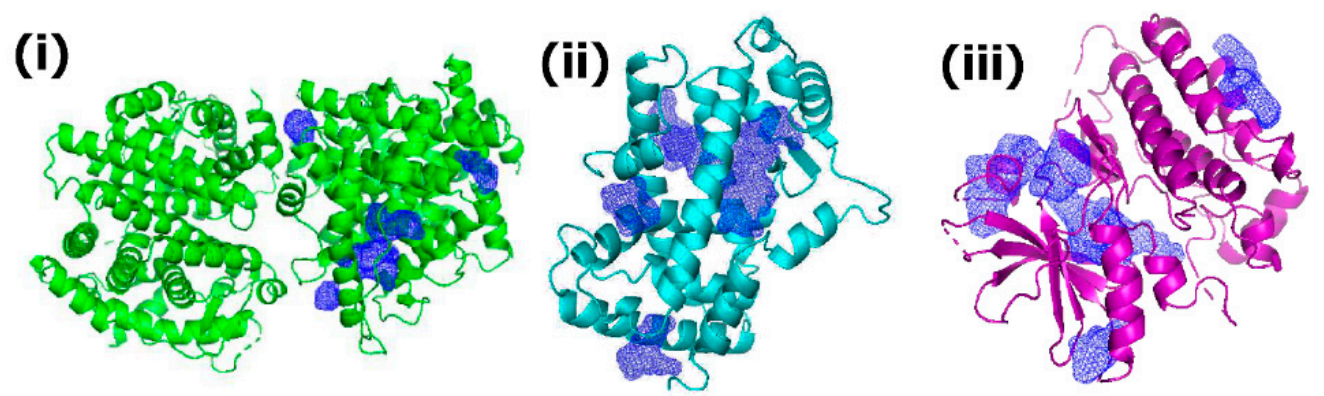

Figure 1. Binding pocket analysis based on POCASA results (i) ER $\alpha$; (ii) PPAR- $\alpha$; (iii) EGFR. Shaded blue regions indicate binding pockets.

For PPAR- $\alpha$, the pocket number 1 had a volume of $165 \AA$ and a VD of 1447. Pockets 2 and 3 had volumes of $196 \AA$ and $76 \AA$ and VD of 1406 and 204, respectively. In previous studies, molecular docking of ligands with PPAR- $\alpha$ showed binding occurred in mostly hydrophobic pockets and interacted with Tyr314, Tyr464, Ser280, and His440 [53]. The POCASA results for EGFR showed that pocket number 180 was ranked number 1 and had a volume of $428 \AA$ and a volume density of 1954 .

\subsection{Molecular Docking Studies}

Receptor-ligand molecular docking studies were conducted using Autodock Vina and FireDock. It has been shown that investigating binding affinity using multiple docking methods improves the reliability of results [54]. The purpose of these studies was to determine the optimal binding affinity upon docking of each of the peptides and peptidepolyphenol conjugates with ER- $\alpha$, EGFR, and PPAR- $\alpha$. Binding affinities obtained from Autodock Vina between peptides or peptide-conjugates and receptors are shown in Table 4, while those obtained from FireDock are shown in Supplementary Information Table S1.

From the data obtained from Autodock Vina, for ER- $\alpha$, RMAMut1 (rosmarinic acid conjugated to Mutation 1 peptide); CGAMut4 (chlorogenic acid conjugated to Mutation 4 peptide), and GLAMut4 (gallic acid conjugated to Mutation 4 peptide) were found to have the highest binding affinities. According to FireDock results, the highest global energies were found to be for GLAMut4, followed by CSAMut2 (carnosic acid conjugated to Mutation 2 peptide) and CGAMut3 (chlorogenic acid conjugated to Mutation 3 peptide). These results indicate that conjugating with certain polyphenols likely increases the binding affinity of the ligands toward ER- $\alpha$. For PPAR- $\alpha$, the binding affinities were found to be relatively lower than the results obtained for ER- $\alpha$. This is plausible due to differences in the binding pockets and their interactions with each of the ligands. Nevertheless, the highest binding affinity for PPAR- $\alpha$ according to Autodock Vina was seen for RMAMut1, followed closely by GLAMut3 and RMAPep. Based on FireDock results, the highest global energy was found to be for CSAMut1, followed by CSAMut3 and GLAMut1. For the EGFR receptor, based on Autodock Vina results, the highest binding affinity was observed for pep at $-9.5 \mathrm{kcal} / \mathrm{mol}$, though this was lower than that observed for the ER- $\alpha(-10.0 \mathrm{kcal} / \mathrm{mol})$. The second-highest binding affinity was observed for RMAMut1, followed by CSAMut3. As per FireDock results, the highest global binding energy was found to be for RMAMut4 followed by CSAMut 3 and GLAMut3. For PPAR- $\alpha$, the point mutation of Tyr in position 1 to Phe improved binding affinity from $-8.9 \mathrm{kcal} / \mathrm{mol}$ to $-9.1 \mathrm{kcal} / \mathrm{mol}$ according to Autodock Vina results, whereas very marginal reduction was observed according to FireDock ( -49.68 to $-49.55 \mathrm{kcal} / \mathrm{mol}$ ), indicating that Mut1 may potentially exhibit high binding affinity for PPAR- $\alpha$. Mut 4 and Mut3 exhibited the second and third highest global binding energy according to FireDock and a high binding affinity according to Autodock Vina as well, indicating that these peptides may have a fairly high binding affinity with PPAR- $\alpha$. Thus, while there were some similarities in the results obtained from FireDock and AutoDock Vina, the results overall showed a selection of different compounds for each of the receptors. Given the variance in results based on the individual analysis of global energy as seen in 
the case of FireDock or estimate of free binding energy ( $\mathrm{kcal} / \mathrm{mol}$ ), as seen in Autodock Vina, it is indicative that the software had dissimilarities between best proposed candidates. This is likely due to differences in the docking algorithms [55]. FireDock provides results from the global binding energy and takes into account electrostatic, Van der Waals, H-bond, and atomic contact energies. Autodock Vina uses a combination of knowledge-based and empirical scoring functions and is the summation of $\Delta \mathrm{G}$ gauss, $\Delta$ Grepulsion, $\Delta \mathrm{GHbond}$, $\Delta$ Ghydrophobic, and $\Delta$ Gtors, which is proportional to the number of rotatable bonds [56]. Overall, based on both docking programs, our results indicated that the conjugates, as well as the peptides, had moderate to good binding affinities, with relatively higher binding affinities observed for ER- $\alpha$ and EGFR receptors compared to PPAR- $\alpha$.

Table 4. Binding Affinities (kcal/mol) of Peptide, mutants and Peptide-Polyphenol Conjugates for ER- $\alpha$; PPAR- $\alpha$, and EGFR using Autodock Vina.

\begin{tabular}{|c|c|c|c|c|c|}
\hline \multicolumn{6}{|c|}{ ER- $\alpha$} \\
\hline $\begin{array}{l}\text { Peptide Sequence } \\
\text { Conjugated }\end{array}$ & Carnosic Acid & Chlorogenic Acid & Gallic Acid & Rosmarinic Acid & $\begin{array}{l}\text { Neat Peptide } \\
\text { (Unconjugated) }\end{array}$ \\
\hline YHWYGYTPQN (pep) & -8.7 & -9.8 & -8.8 & -10.3 & -10.0 \\
\hline FHWYGYTPQN (Mut1) & -7.8 & -10.2 & -9.0 & -8.7 & -8.6 \\
\hline YIWYGYTPQN (Mut2) & -7.8 & -9.6 & -9.5 & -8.6 & -8.6 \\
\hline YHWYGYTHQN (Mut3) & -10.0 & -9.1 & -8.1 & -8.2 & -10.0 \\
\hline YHWYGYTPQD (Mut4) & -9.0 & -10.3 & -10.2 & -9.4 & -9.1 \\
\hline \multicolumn{6}{|c|}{ PPAR- $\alpha$} \\
\hline $\begin{array}{c}\text { Peptide Sequence } \\
\text { Conjugated }\end{array}$ & Carnosic Acid & Chlorogenic Acid & Gallic Acid & Rosmarinic Acid & $\begin{array}{c}\text { Neat Peptide } \\
\text { (Unconjugated) }\end{array}$ \\
\hline YHWYGYTPQN (pep) & -7.1 & -8.1 & -8.3 & -9.2 & -8.9 \\
\hline FHWYGYTPQN (Mut1) & -6.5 & -7.0 & -8.3 & -9.5 & -9.1 \\
\hline YIWYGYTPQN (Mut2) & -6.2 & -7.1 & -9.2 & -5.4 & -7.6 \\
\hline YHWYGYTHQN (Mut3) & -6.5 & -7.2 & -7.4 & -7.6 & -8.9 \\
\hline YHWYGYTPQD (Mut4) & -7.0 & -6.8 & -8.6 & -8.3 & -9.1 \\
\hline \multicolumn{6}{|c|}{ EGFR } \\
\hline $\begin{array}{c}\text { Peptide Sequence } \\
\text { Conjugated }\end{array}$ & Carnosic Acid & Chlorogenic Acid & Gallic Acid & Rosmarinic Acid & $\begin{array}{c}\text { Neat Peptide } \\
\text { (Unconjugated) }\end{array}$ \\
\hline YHWYGYTPQN (pep) & -8 & -8.5 & -8.3 & -8.4 & -9.5 \\
\hline FHWYGYTPQN (Mut1) & -8.3 & -8.3 & -7.7 & -9.1 & -7.7 \\
\hline YIWYGYTPQN (Mut2) & -7.2 & -8.2 & -7.7 & -8.4 & -8.7 \\
\hline YHWYGYTHQN (Mut3) & -8.6 & -7.4 & -7.4 & -7.4 & -8.0 \\
\hline YHWYGYTPQD (Mut4) & -8.1 & -7.5 & -8.4 & -8.8 & -8.3 \\
\hline
\end{tabular}

\subsection{Binding Interaction Analysis with PLIP}

\subsubsection{Interactions with Estrogen Receptor}

Following docking, the PLIP interface was used to study the interactions. Results for ER- $\alpha$ with peptides are shown in Figure 2 and Supplementary Information Table S2. As shown in Figure 2a, and Supplementary Information Table S2a, Pep exhibited eighteen hydrogen bonds and eight hydrophobic interactions with ER- $\alpha$. Additionally, a $\pi$-stacking interaction between Tyr537A and Tyr6 of Pep, as well as salt bridges between His516D and Lys520D and the carboxy-terminal of Pep, were observed. Tyr537 is a critical residue in the binding pocket of ER- $\alpha$ that plays an important role in hormone binding and DNA binding and is essential for the conformational changes that occur within the receptor to 
activate downstream transcription processes [57]. This indicates that Pep interacts within the same region.

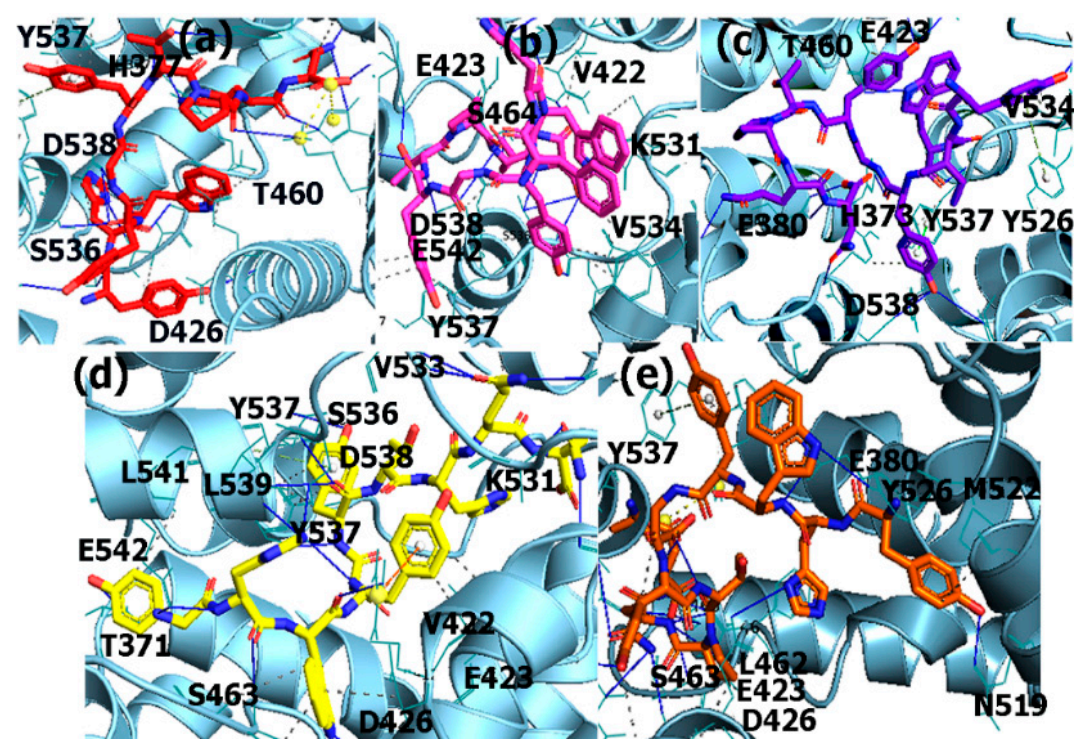

Figure 2. PLIP results with ER- $\alpha$ for (a) Pep; (b) Mut1 (c) Mut2 (d) Mut3 (e) Mut4.

For the Mutation 1 peptide (Mut1), which involved altering the N-terminal Tyr to Phe, results are shown in Figure $2 \mathrm{~b}$ and Supplementary Information Table S2b. Fifteen H-bonds and eleven hydrophobic interactions were observed; an H-bond with Asp426 that had been observed for the original peptide was eliminated, which explains the reduction in binding affinity upon mutating $Y$ to $F$. No $\pi$-stacking or salt bridges occurred, due to a slight change in orientation of the ligand within the receptor binding site.

The mutation of His to Ile (Mut2) resulted in fourteen H-bonds and five hydrophobic interactions with ER- $\alpha$, as seen in Figure 2c and Supplementary Information Table S2c. Interestingly, with the exception of one H-bond with Asp426D, H-bonds with different residues of ER- $\alpha$ were formed compared to those with Pep. Some hydrophobic interactions seen for Pep were maintained, including those with Glu423D, Thr460B, and Tyr537B, while new interactions were formed with Val534A and Tyr537A of ER- $\alpha$. Two $\pi$-stacking interactions occurred between Tyr residues of ER and Mut2.

For Mut3, (Figure 2d and Supplementary Information Table S2d), eighteen H-bonds and eleven hydrophobic interactions occurred. New H-bonds formed with residues, including Thr371B, Arg412D, Glu419D, Met528A, Lys531A, Val533A, indicating a slight change in position of the Mut3 peptide within the receptor compared to Pep. Certain hydrophobic interactions were maintained, including those with Glu423D, Ser463B, and Tyr537B, while new hydrophobic interactions formed with several residues including Val422D, Asp426D, Leu462B, Tyr526A, Tyr537A, and Leu541A. A new $\pi$-cation interaction was observed between Tyr4 of Mut 3 and Arg412D of ER- $\alpha$ and the $\pi$-stacking interaction between Tyr537A of ER- $\alpha$ and Tyr6 of the peptide was conserved and is likely responsible for the higher binding compared to Mut2.

For Mut4 (Figure 2e and Supplementary Information Table S2e), eleven hydrophobic interactions and fourteen hydrogen bonds were formed between Mut 4 and ER. Hydrophobic interactions were all formed with different residues compared to Pep. A H-bond was observed with Asp538A, as was seen for Pep, while all other H-bonds formed with new residues of ER- $\alpha$. $\pi$-stacking was observed between Tyr537B of ER and Tyr4 of the Mut4 peptide and a $\pi$-cation interaction occurred between Lys520C of ER and the N-terminal Tyr of Mut4. Three salt bridges were formed between Arg515A, His516C, and Lys520C of ER and the C-terminal Asp of Mut4.

These results indicate that the mutated peptides showed comparable binding interactions with ER- $\alpha$, though for some there was a slight reduction (Mut1 and Mut2) compared 
to Pep. Mut3 showed similar binding affinity to Pep, while Mut4 showed a very slight reduction. Further, new interactions were formed between some of the mutated peptides and ER- $\alpha$, which could potentially enhance the peptides' selectivity and stability in the receptor binding site.

After examining the binding interactions of the peptides, we then examined the binding interactions of the polyphenol-peptide conjugates. The interactions are shown in Supplementary Information Table S3 and Figure 3.

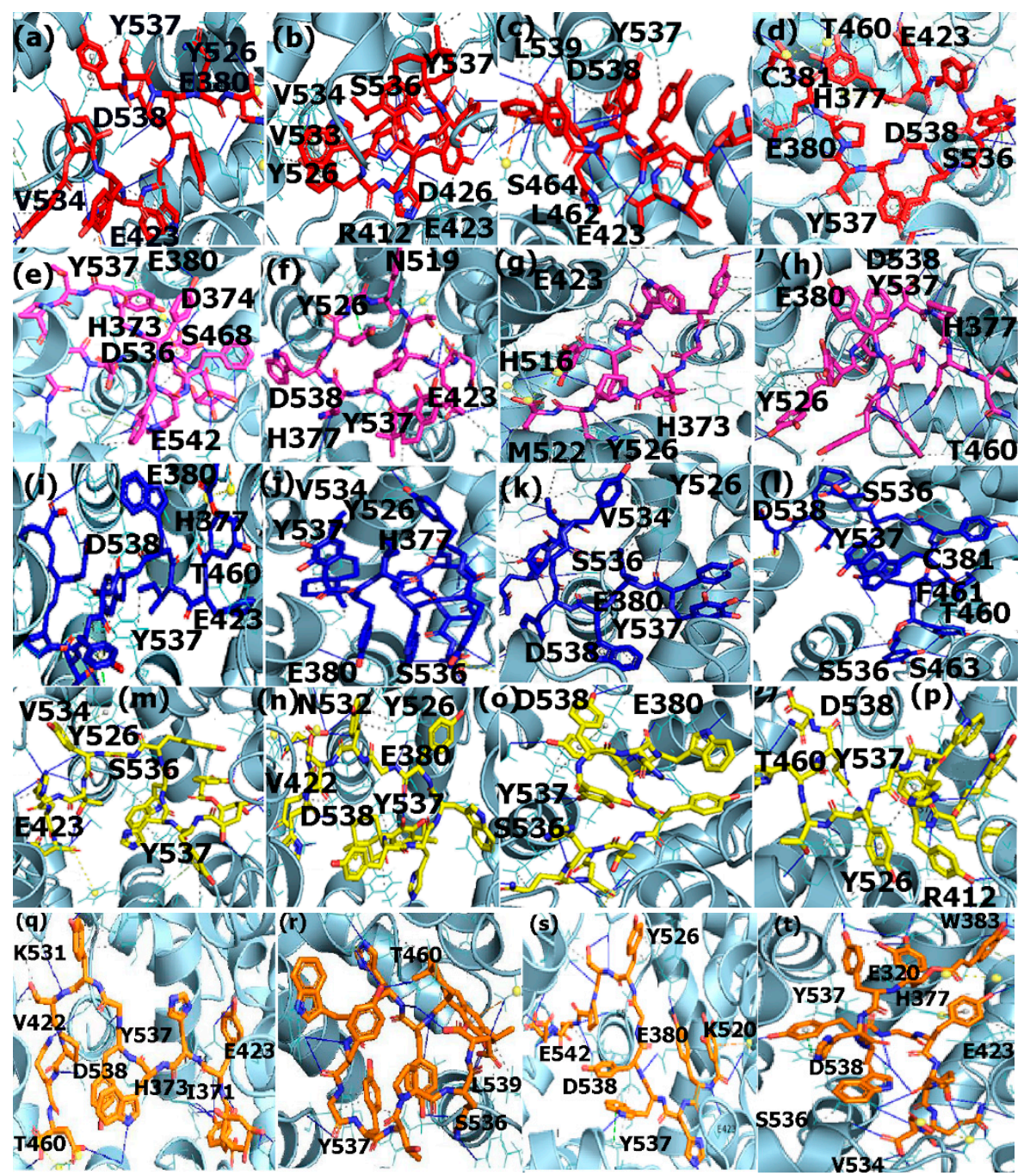

Figure 3. PLIP results for polyphenol peptide conjugates bound to ER $\alpha$ (a) CGA-Pep; (b) CSA-Pep (c) GLA-Pep (d) RMA-Pep (e) Mut1-CGA; (f) Mut1-CSA; (g) Mut1-GLA; (h) Mut1-RMA; (i) Mut2CGA (j) Mut2-CSA; (k) Mut2-GLA; (1) Mut2-RMA; (m) Mut3-CGA; (n) Mut3-CSA; (o) Mut3-GLA; (p) Mut3-RMA; (q) Mut4-CGA; (r) Mut4-CSA; (s) Mut4-GLA; (t) Mut4-RMA.

In a recent study, comparative molecular binding studies of ER- $\alpha$ models with their native ligand estradiol, the drug genistein, and homoisoflavones demonstrated that homoisoflavones exhibited comparable binding affinity for ER- $\alpha$ as that of estradiol and genistein, indicating their potential applications as ER- $\alpha$ ligands. Several residues, including Leu339, Leu343, Phe356, Phe475, Glu305, and Ile373, were particularly implicated in binding interactions [58]. Various polyphenols have also been shown to act as ER- $\alpha$ ligands and this property is implicated in their anti-tumorigenic properties in ER positive cancers [59]. Thus, we hypothesized that the designed polyphenol-peptide conjugates may also display favorable binding interactions with $\mathrm{ER} \alpha$. 
The results obtained after conjugation with polyphenols are shown in Figure 3. Results for CGAPep are shown in Supplementary Information Table S3a and Figure 3a. Extensive hydrophobic interactions (ten) and hydrogen bonds (twenty) occurred. $\pi$-stacking interactions occurred between Tyr526A and Tyr537A of ER- $\alpha$ and CGAPep. A $\pi$-cation interaction also occurred between Arg412D of ER- $\alpha$ and CGAPep and a salt bridge was observed between His516D and the C-terminal of CGAPep. The results obtained for CSAPep are shown in Supplementary Information Table S3b and Figure 3b. As shown, conjugation with carnosate led to nine hydrophobic interactions and only twelve hydrogen bonds formed. Some hydrophobic interactions that were seen for Pep were eliminated, including those with Glu423D, Thr460B, and Met522B, while new ones were formed with Asp426D, Tyr526A, Asn532A, and Val534A. New hydrogen bonds were formed in several locations, including Arg412D, Glu423D, Thr460B, Asn532A, and Val533A. While three $\pi$-stacking interactions occurred with Tyr526A of ER, salt bridges were absent. The GLA-Pep conjugate (Supplementary information Table S3c and Figure 3c) formed sixteen hydrogen bonds and seven hydrophobic interactions were observed in addition to one $\pi$-cation interaction. New hydrophobic interactions were formed with Thr465B, Tyr537A, Leu539A, Leu541A, and Glu542A. Several hydrogen bonds that were observed for Pep were eliminated, including those with Glu380B, Asp426D, Ser463B, His516D, Ser518B, and others; however, new H-bonds were observed with Arg412D, Glu423D, Phe461B, and Asn532A. For RMAPep (Figure 3d, and Supplementary Information Table S3d), eighteen hydrogen bonds and ten hydrophobic interactions were observed, and an additional $\pi$-stacking interaction formed between Tyr526A and RMAPep. Some hydrophobic interacting residues seen for Pep were observed, while new residues included Val422D, Leu462B, and Tyr526A. Several changes in hydrogen bonding positions were also observed, including new H-bonds with Cys381B, Trp383B, Arg412D, Met427D, Thr460B, Asn532A, Tyr537A, and Tyr537B.

We next explored the binding interactions with the Mut1-polyphenol conjugates. The chlorogenate-Mut1 (CGAMut1) results are shown in Supplementary Information Table S3e and Figure 3e. Hydrogen bonds with Arg412D, Asn532A, Ser536A, and Asp538A were still observed, while others were formed with new residues, including Thr371B, Asp374B, Glu380A, Glu380B, Trp383A, Asp426D, and Val534A, among other residues. Hydrophobic interactions were still seen with Glu423D, Tyr426A, Val534A, Tyr537A, Tyr537B, and Asp538A, while new hydrophobic interactions were seen with Asp426D, Val534A, and Glu542A. $\pi$-stacking occurred with Tyr526A and Tyr537B, respectively. A $\pi$-cation interaction occurred between His373B of ER- $\alpha$ and CGAMut1. Results for CSAMut1 are shown in Supplementary Information Table S3f and Figure 3f. This conjugate exhibited six hydrophobic interactions and thirteen hydrogen bonds. Hydrophobic interactions with Tyr526A, Tyr537B, Asp538A, Val422C, and Lys531B were observed. One $\pi$-stacking interaction and one salt bridge occurred. For the gallate-Mut1 (GLAMut1) conjugate, nine hydrophobic interactions and thirteen H-bonds occurred, as shown in Supplementary Information Table S3g and Figure $3 g$. The H-bonds were observed with different residues compared to those seen for neat Mut1, indicating a change in position of the ligand within the receptor binding site upon conjugation with gallate. Hydrophobic interactions with Tyr526A and Tyr537B were still seen, while all others were formed with different residues, including Glu423C, Thr460A, and Met522A. $\pi$-stacking occurred with Tyr526B and salt bridges occurred with His516C and Lys520C. RMAMut1 (Figure 3h and Supplementary Information Table S3h) led to the formation of twenty hydrogen bonds and eleven hydrophobic interactions with ER- $\alpha$. Many new hydrogen bonds were formed, including with His373B, Glu380A, Trp383B, Asn519B, Lys520D, Leu525A, Tyr526B, Met528A, Tyr537A, and Asp538B. Several new hydrophobic interactions occurred with Asp426D, Thr460B, and Val534B. Additionally, a $\pi$-stacking interaction was observed with Tyr526A.

PLIP studies were next performed with Mut2 conjugated with all four polyphenols. The CGAMut2 conjugate (Supplementary Information Table S3i and Figure 3i) exhibited marked improvement in binding affinity. In total, seventeen H-bonds and eleven hydrophobic interactions, as well as the formation of a $\pi$-stacking interaction, a $\pi$-cation 
interaction, and a salt bridge was observed. New hydrogen bonds were observed with Glu423C, Asp426C, Thr460A, Phe461B, His516C, Asn519A, Lys520D, Tyr526B, Ser536A, and Asp538A. New hydrophobic interactions were seen with Glu380B, Asp426C, Asn519A, Lys520C, Tyr526A, and Tyr526B. For CSAMut2, the results are shown in Figure 3j and Supplementary Information Table S3j. Six hydrophobic interactions and sixteen H-bonds were observed. New hydrophobic interactions were seen with Tyr526A, while new H-bonds occurred with Trp383A, Arg412C, Glu423C, Thr460A, Lys520C, Lys531B, Ser536B, and Leu539B. A salt bridge was also observed with Arg412C. The results obtained for GLAMut2 are shown in Supplementary Information Table S3k and Figure 3k. Additional hydrophobic interactions (a total of eleven) and H-bonding (a total of nineteen) compared to neat Mut2 were seen. A $\pi$-stacking interaction with Tyr537A was also seen. New hydrophobic interactions include those with Glu380A, Val422D, Tyr526A, Lys531A, Asn532A, and Leu539A, while new H-bonds included those with Phe461B, Ser464B, His516C, Lys520C, Tyr526A, Met528A, and Ser536A. For RMAMut2 (Figure 31 and Supplementary Information Table S31), nineteen hydrogen bonds and five hydrophobic interactions occurred; a salt bridge was observed with His377A. New H-bonds included those with Cys381B, Tyr459B, Phe461B, Leu462A, Leu462B, Ser463B, and His516D. New hydrophobic interactions occurred with Asp426D, Tyr526B, and Asp538A.

CGAMut3 showed a slight decrease in H-bonding (fifteen total); fewer hydrophobic interactions (six total) were observed compared to neat Mut3, while $\pi$-stacking occurred with Tyr526A and Tyr526B in addition to a salt bridge with His377B (Supplementary Information Table S3m and Figure 3m). Compared to Mut3, hydrogen bonds with Thr371B, Glu419D, Met528A, Val533A, Val534A, Tyr537A, Leu539A, and Glu542A were eliminated, while several other new $\mathrm{H}$-bonds were observed. New hydrophobic interactions were also seen. In the case of CSAMut3, twelve hydrogen bonds and thirteen hydrophobic interactions occurred and $\pi$-stacking and $\pi$-cation interactions were not maintained, while a salt bridge was formed with Arg412D, as shown in Figure 3n and Supplementary Information Table S3n. Several hydrogen bonds seen for neat Mut3 were not observed, including those with Thr371B, Glu419D, Leu462B, Met528A, Lys531A, and Glu542A. New hydrophobic interactions formed included those with His377A, Val534A, and Asp538A. For GLAMut3 (Figure 3o and Supplementary Information Table S3o), four hydrophobic interactions and sixteen H-bonds occurred, while $\pi$-stacking and a salt bridge were observed. Hydrophobic interactions were seen with Tyr537A, Tyr537B, and Leu539B. Several hydrogen bonds disappeared, including those with Thr371B, Glu419D, Asp426D, Leu462B, Met528A, Lys531A, Ser536A, and Leu539A, while new H-bonds were formed with several other residues. The RMAMut3 conjugate showed four hydrophobic interactions and nineteen H-bonds, in addition to $\pi$-stacking interactions and a salt bridge (Supplementary Information Table S3p and Figure 3p). Hydrophobic interactions were observed with Glu380A, Val534A, Tyr537A, and Tyr537B. New H-bonds were formed with His373A, His373B, Glu380B, Arg412C, Val422C, Glu423C, Thr460B, His516C, and Lys520C, among other residues. The significant changes in the positions of receptor-ligand interactions indicate a change in the position of RMAMut3 within the binding pocket compared with Mut3.

PLIP studies were then carried out with Mut4-conjugated polyphenols. For CGAMut4, eighteen hydrogen bonds and twelve hydrophobic interactions were observed, while two salt bridges were formed between His377A (Supplementary Information Table S3q and Figure 3q). New H-bonds formed include those with Thr371B, Asp374B, Arg412C, and Ser536B. New hydrophobic interactions include those with Val422C, Lys531B, Asp538B, Leu541A, and Glu542A. No $\pi$-stacking or $\pi$-cation interactions were formed. The CSAMut4 conjugate (Supplementary Information Table S3r and Figure 3r) showed fourteen H-bonds and nine hydrophobic interactions. Nearly all H-bonds observed were formed with different residues compared to those for Mut4 alone; interacting residues include Arg412D, Glu423D, His516D, Lys520D, and Ser536A. New hydrophobic interactions included those with Thr460B, Leu462B, and Leu539A. $\pi$-stacking and salt bridges seen for neat Mut4 were not maintained. A $\pi$-cation interaction was observed with Arg412D. 
For GLAMut4, the conjugate exhibited nineteen H-bonds and eleven hydrophobic interactions (Supplementary Information Table S3s and Figure 3s). New H-bonds were seen with Arg412D, Asp426C, Met427D, Leu462B, Ser463B, His516C, Lys520C, Ser536A, and Glu542A, while new hydrophobic interactions occurred with Glu380A, Val422D, and Asp538A. A $\pi$-stacking interaction was formed with Tyr537B and a $\pi$-cation interaction was seen with His516C. For the RMAMut4 conjugate, additional H-bonding (twenty-two total) was observed and slightly reduced hydrophobic interactions (nine) occurred compared to Mut4 alone (Supplementary Information Table S3t and Figure 3t). New H-bonds included those with Cys381B, Trp383B, Arg412D, Asp426D, His516D, Ser518B, and Asn519B and new hydrophobic interactions included those with Glu380B, Thr460B, and Ser463B. $\pi$-stacking interactions were formed with His516D and Tyr537A, a $\pi$-cation interaction was observed with His377B, and salt bridges occurred with Arg412D and Lys520D.

These results indicated that all of the polyphenol-peptide conjugates, as well as the peptides, interacted with the ER- $\alpha$; furthermore, the conjugation of select peptides with some of the polyphenols enhanced binding. Specifically, the binding interactions of Mut1 was improved by conjugation with gallate, while that of Mut2 improved upon conjugation with all four of the polyphenols. In the case of Mut3, improved binding interactions were observed with CGA, while, for Mut4, conjugation with gallate enhanced interactions. The relatively stronger binding interactions between chlorogenate conjugates and $\mathrm{ER} \alpha$ were likely resultant of the hydrogen bonding ability of CGA. In comparison to CGA, carnosate is relatively hydrophobic due to its extensive ring system, which likely contributed to the weaker binding interactions observed for carnosate conjugates with ER- $\alpha$. It is interesting to note that, among the mutated peptides, Mut3 and Pep had very similar binding interactions, indicating that changing Y-H-W-Y-G-Y-T-P-Q-N to Y-H-W-Y-G-Y-T-H-Q-N did not have a large impact on binding, though His is significantly different in properties from proline. This can be attributed to the fact that both peptides showed an equal number of H-bonding interactions with the ER- $\alpha$, indicating that H-bonding played a key role in overall binding interactions.

\subsubsection{Interactions with PPAR- $\alpha$}

Peroxisome proliferator-activated receptors (PPARs) are members of the nuclear hormone receptor superfamily that are responsible for cellular responses. PPARs are regulators of lipid and carbohydrate metabolism; they also modulate inflammatory responses [60]. Their over-expression has also been implicated in breast cancer. PPARs are activated by a variety of ligands, including cholesterol metabolites, eicosanoids, retinoids, fatty acids, steroids and several antidyslipidemic drugs [61]. In previous studies, the crystal structure of the PPAR- $\alpha$ ligand binding domain (LBD) in complex with a polyphenolic dihydro cinnamate derivative was studied; it was shown that a conserved H-bonding network formed between the cinnamate derivative and the AF2 helix played an important role in forming a stable structure. This study further showed that the LBD of PPAR- $\alpha$ occurred at the C-terminus, composed of 12 helices in an antiparallel helix sandwich, as well as a 3-stranded antiparallel $\beta$-sheet. Ligands generally bind to Tyr437, Tyr446, Ser280, and His440 of the PPAR- $\alpha$ ligand-binding motif [62]. In another study, molecular docking of several benzenesulphonamide derivatives with PPAR- $\alpha$ revealed residues including Met355, Met330, Met220, Val332, Val324, Ile317, Leu331, Leu321, Ser280, Asn219, Gly335, and Cys276, among others, that were involved in interactions [63].

The PLIP results showing the residues of the PPAR- $\alpha$ receptor involved in binding interactions with the peptides are shown in Figure 4 and Supplementary Information Table S4. The binding interactions for Pep with PPAR- $\alpha$ showed ten hydrophobic interactions and twelve hydrogen bonds, shown in Figure 4a and Supplementary Information Table S4a. Met220A, Ile241A, Val281A, Leu321A, and Val332A were involved in hydrophobic interactions and Ala256A, His274A, Leu331A, Gly335A, Ala455A, and His457A were involved in H-bonding, while Lys257A, Thr279A, Ala333A, and Tyr334A were involved in both hydrophobic interactions and H-bonding. 




Figure 4. PLIP results for each of the peptides bound to PPAR $\alpha$ receptor. (a) Original peptide; (b) Mut1 (c) Mut2 (d) Mut3 (e) Mut4.

For Mut1 (Figure 4b and Supplementary Information Table S4b), thirteen hydrophobic interactions, twelve $\mathrm{H}$-bonds, and a $\pi$-stacking interaction with Tyr334A were observed. New hydrophobic interactions were observed with Thr283A, Met320A, and Val324A, while new H-bonds occurred with Leu254A, Thr283A, and Thr285A. For Mut2, extensive hydrogen bonding (fourteen) and hydrophobic interactions (eleven) were observed compared with Pep, and a $\pi$-stacking interaction with Tyr334A was again observed, as shown in Figure 4c and Supplementary Information Table S4c. New hydrogen bonds occurred with Tyr214A, Lys222A, Leu254A, and Ser323A, while new hydrophobic interactions were seen with Glu282A, and Met320A. Mut3 (Figure 4d and Supplementary Information Table S4d) exhibited thirteen hydrophobic interactions and sixteen H-bonds. New hydrophobic interactions occurred with Val255A, Thr283A, Thr285A, Glu286A, Glu289A, and Val324A, while new H-bonds occurred with Asn217A, Asn219A, Met220A, Glu282A, Thr283A, Glu286A, and Glu289A. Mut4 (Figure 4e and Supplementary Information Table S4e), displayed ten hydrophobic interactions and ten hydrogen bonds. New hydrophobic interactions were observed with Lys224A, Asn236A, Leu254A, and Val324A and new H-bonds were seen with Lys224A, Ser234A, Thr253A, and Thr283A. A salt bridge was formed with Lys224A.

PLIP interactions of the peptide-polyphenol conjugates and PPAR $\alpha$ were next examined as shown in Figure 5 and Supplementary Information Table S5. The CGAPep conjugate displayed seventeen $\mathrm{H}$-bonds and fourteen hydrophobic interactions as well as two $\pi$-stacking interactions (Figure 5a and Supplementary Information Table S5a). New hydrogen bonds formed included those with Lys216A, Asn219A, Leu254A, Cys278A, Thr279A, Glu282A, Thr285A, and Glu696B, while new hydrophobic interactions were seen with Val255A, Leu258A, Glu282A, Ile339A, and Leu693B compared to Pep. As seen in Figure $5 \mathrm{~b}$ and Supplementary information Table S5b, eight hydrogen bonds and nine hydrophobic interactions were observed for CSAPep, while a $\pi$-stacking interaction occurred with Tyr334A of PPAR- $\alpha$. Hydrogen bonds were still observed with Thr279A and Tyr334A, while all others were seen with new residues, including Lys224A, Glu282A, and Asn336A. All hydrophobic interactions, except for two with Tyr334A, were formed with new residues, including Val255A, Val281A, Glu282A, Thr285A, Glu286A, and Glu289A. The GLAPep conjugate displayed fourteen hydrogen bonds and thirteen hydrophobic interactions, as seen in Figure 5c and Supplementary Information Table S5c. New hydro- 
gen bonds included those with Asn219A, Lys224A, Ser334A, Asn236A, Ile272A, Cys275A, Glu282A, and Asn336A, while new hydrophobic interactions were observed with Asn219A, Lys224A, Ile228A, Asn236A, Leu254A, Val255A, Glu282A, and Ile339A. Conjugation with rosmarinate (RMAPep) improved the binding affinity of Pep for PPAR- $\alpha$. As shown in Figure 5d and Supplementary Information Table S5d, seventeen hydrophobic interactions and fourteen hydrogen bonds were observed. Hydrogen bonds observed included those with Asn219A, Met220A, Cys275A, Glu282A, Thr285A, Glu286A, and Arg692B, while new hydrophobic interactions included those with Leu254A, Val255A, Ile317A, Phe318A, Met320A, Val324A, and Leu693B. Additionally, two $\pi$-stacking interactions were formed with Tyr334.

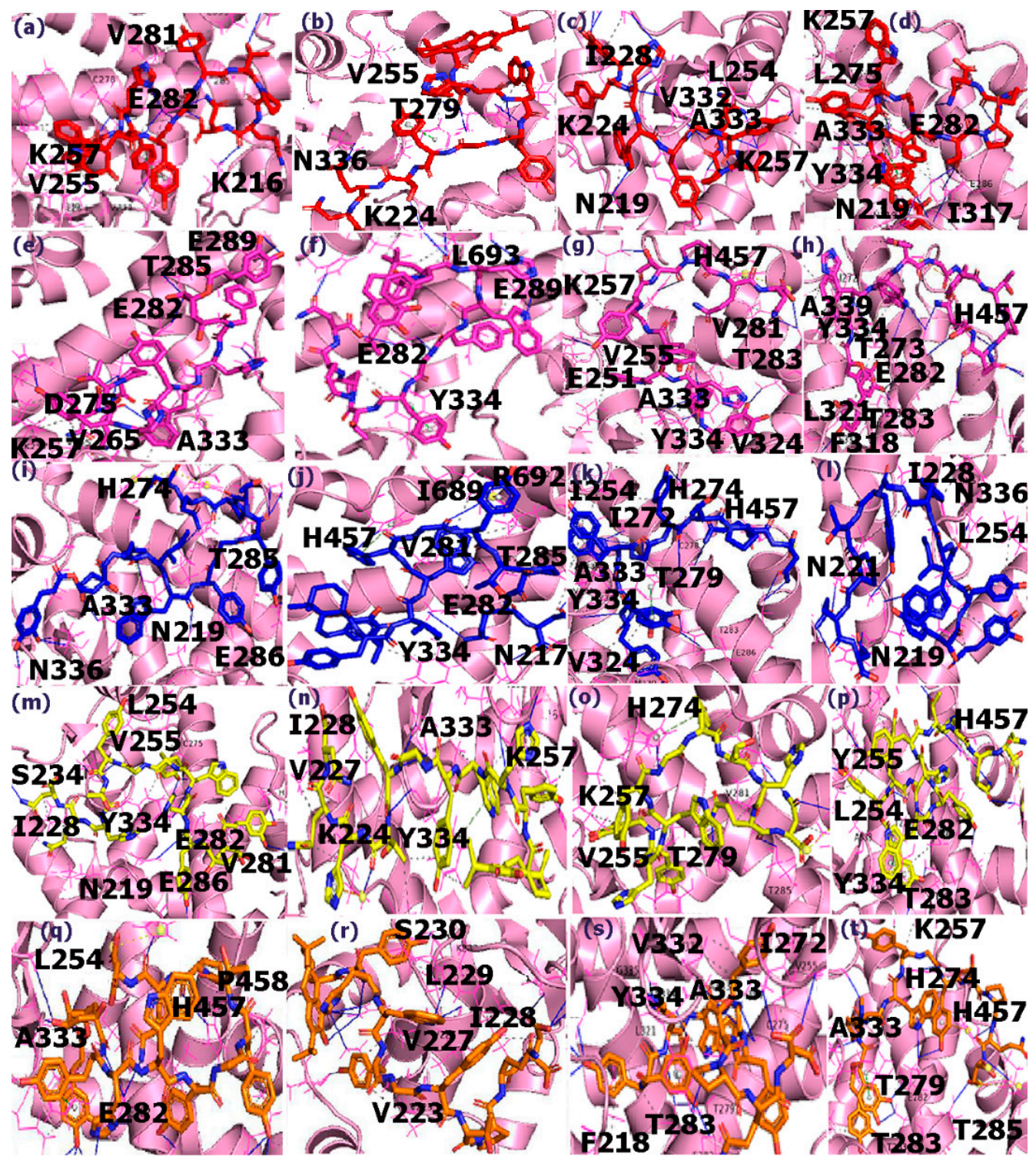

Figure 5. PLIP results for polyphenol peptide conjugates bound to PPAR- $\alpha$ receptor. Pep conjugated with (a) cholorogenate; (b) carnosate (c) gallate (d) rosmarinate. Mut1-conjugated with (e) cholorogenate; (f) carnosate; (g) gallate; (h) rosmairnate. Mut2-conjugated with (i) chlorogenate (j) carnosate; (k) gallate; (1) rosmairnate. Mut 3-conjugated with (m) cholorogenate; (n) carnosate; (o) gallate; (p) rosmairnate; Mut4-conjugated with (q) cholorogenate; (r) with carnosate; (s) gallate; (t) rosmairnate.

The conjugate CGAMut1 (Figure 5e and Supplementary Information Table S5e) showed eleven $\mathrm{H}$-bonds and fourteen hydrophobic interactions. New H-bonds were observed with 
Asn219A, Cys275A, Glu282A, and Glu289A, while new hydrophobic interactions occurred with Leu254A, Val255A, Leu258A, Glu282A, Thr285A, Glu286A, and Glu289A. As shown in Figure $5 f$ and Supplementary Information Table S5f, carnosate-Mut1 (CSAMut1) conjugate displayed eight hydrophobic interactions and nine H-bonds, while the $\pi$-stacking was not maintained. Several hydrophobic interactions seen for Mut1 alone were eliminated, while new ones formed. New H-bonds were also observed with Glu282A, Glu289A, Arg692B, and Glu696B. A $\pi$-stacking interaction was observed with Tyr334A. The GLAMut1 conjugate exhibited thirteen hydrophobic interactions and ten hydrogen bonds as shown in Figure $5 \mathrm{~g}$ and Supplementary Information Table S5g. New hydrophobic interactions were observed with Leu247A, Glu251A, Val255A, and Val281A. A new hydrogen bond was observed with Leu331A. A salt bridge also occurred with His457A. The RMAMut1 (Figure 5h and Supplementary information Table S5h), formed fifteen H-bonds and twenty hydrophobic interactions. New H-bonds were seen with Ser280A, Glu282A, Met320A, His440A, and Arg692B. New hydrophobic interactions occurred with Leu247A, Val255A, Ile272A, Thr285A, Phe318A, Ile339A, His457A, and Leu693B. $\pi$-stacking was observed between Tyr334 and a $\pi$-cation interaction was shown between His 274 of PPAR- $\alpha$ and Tyr6 of RMAMut1.

The CGAMut 2 conjugate (Figure $5 \mathrm{i}$ and Supplementary information Table S5i), formed eleven hydrophobic interactions, with new ones occurring with Leu254A, Val281A, Thr285A, Glu286A, Glu289A, and Leu459A. Twelve hydrogen bonds occurred, with new ones including those with Asn219A, Asn236A, Thr279A, Asn336A, and Arg692B. $\pi$-stacking with Tyr334A and salt bridges with His257A and His457A were also observed. CSAMut2 on the other hand, as shown in Figure $5 \mathbf{j}$ and Supplementary information Table S5j, showed only six hydrophobic interactions and eight hydrogen bonds. $\pi$-stacking with His457A and a $\pi$-cation interaction with Arg692B were again seen. GLA-Mut2 (Figure 5k and Supplementary information Table S5k), showed increased H-bonds (fourteen hydrogen bonds) and seventeen hydrophobic interactions. New H-bonds were seen with Met220A, Ile272A, His274A, Cys276A, Cys278A, Thr285A, Glu286A, and Leu331A. New hydrophobic interactions were observed with Leu247A, Leu254A, Val255A, Ile272A, Val324A, and Ile339A. $\pi$-stacking was observed with Tyr334, while a salt bridge was formed with His457. RMAMut2 (Figure 51 and Supplementary information Table S5l), showed however showed only nine hydrophobic interactions and eight $\mathrm{H}$-bonds.

CGAMut3 (Figure 5m and Supplementary Information Table S5m), showed ten hydrophobic interactions including new ones with Lys224A, Ile228A, Leu254A, Val281A, and Glu282A. Eleven hydrogen bonds were seen, new ones occurring with Ser234A, Cys257A, Asn226A, His457A, and Arg692B. The CSAMut3 conjugate shown in Figure 5n and Supplementary Information Table S5n, exhibited twelve hydrophobic interactions, including those with Asn219A, Lys224A, Val227A, Ile228A, Asn236A, and Lys257A. Eight H-bonds were seen, $\pi$-stacking was once again seen with Tyr334A, and a $\pi$-cation interaction with Lys224A was observed. GLAMut3 (Figure 5o and Supplementary Information Table S5o), showed only six H-bonds with His274A, Thr279A, Glu282A, Thr285A, His457A, and Arg692B. Of the total of nine hydrophobic interactions, new ones were seen with Lys257A, Thr279A, Val281A, Glu282A, His457A, and Leu 693B. $\pi$-stacking was observed with His274A and a salt bridge was observed with Arg692B. RMAMut3 (Figure 5p and Supplementary Information Table S5p) displayed eleven hydrophobic interactions, including new ones with Leu254A, Lys257A, Leu258A, Thr279A, Val281A, and Glu282A. Thirteen H-bonds were shown, including new ones with Ala250A, Ala256A, Lys257A, Thr283A, Thr285A, His457A, and Arg692B. Three $\pi$-stacking interactions were seen with Tyr334A and one with His457A, while a salt bridge was formed with Arg692B.

The CGAMut4 conjugate (Figure 5q and Supplementary Information Table S5q) displayed lesser hydrophobic interactions (seven) with Met220A, Thr279A, Val281A, Tyr334A, Pro458A, and Ile689B but higher H-bonds (sixteen), including new ones with Asn219A, Met220A, Leu254A, Glu282A, Thr285A, Glu286A, His457A, and Arg692B. $\pi$-stacking with Tyr334A was shown, as was a salt bridge with His274A. CSAMut4 (Figure 5r and 
Supplementary Information Table S5r) displayed nine hydrophobic interactions and twelve H-bonds, including new hydrophobic interactions with Val223A, Arg226A, Val227A, Ile228A, Leu229A, Lys232A, and Asn336A and new H-bonds with Lys222A, Arg226A, Val227A, Ser230A, Asn236A, and Leu370A. However, for GLAMut4 conjugate (Figure 5s and Supplementary Information Table S5s) increased H-bonds (nineteen) and ten hydrophobic interactions were observed, as was a $\pi$-stacking interaction with Tyr334. New H-bonds included those with Tyr214A, Lys222A, Leu254A, Ala256A, Cys275A, Glu282A, Ser323A, and Gly335A, indicating overall higher interactions. RMAMut4 (Figure $5 \mathrm{t}$ and Supplementary Information Table S5t) showed relatively less (seven) hydrophobic interactions, including new ones with Lys257A, Val332A, and Ile689B. Thirteen H-bonds were still formed, including new ones with Leu254A, Ala256A, Glu282A, Thr285A, His457A, and Arg692B. A $\pi$-stacking interaction with Tyr334A, a $\pi$-cation interaction with His457A, and two salt bridges with Arg692B were also formed.

Overall, binding studies conducted showed that highest binding free energies according to FireDock were observed for CSAMut3, CGAMut4, and RMAMut3 as well as the neat Mut3 peptide. According to Autodock Vina, however, the results showed that the highest binding affinities were for Mut1, Mut2, RMAPep, GLAMut2, and RMAMut1. Several residues were significantly implicated in binding with peptides and peptide-polyphenol conjugates as predicted according to PLIP, including Thr279A, Thr285A, Tyr334A, Leu254A, Leu321A, Ile228A, Ala333A, Val255A, and Val281A, attesting to the relative hydrophobicity of the binding site. Though the binding sites seen for peptides and conjugates varied, molecules bound in or near the ligand binding domain found in other studies, as indicated by the overlap with residues such as Thr279A, Leu254A, Leu321A, among other interacting residues [64].

\subsubsection{Interactions with EGFR}

PLIP results for each of the peptides docked with EGFR are shown in Figure 6 and detailed interactions are indicated in Supplementary Information Table S6. Eight hydrophobic interactions and nine hydrogen bonds were predicted, according to PLIP as shown in Figure 6a and Supplementary Information Table S6a. Mut1, as indicated in Figure 6b and Supplementary Information Table S6b, showed comparatively lower (six) hydrophobic interactions and six $\mathrm{H}$-bonds, as well as a salt bridge with Lys745A. Hydrophobic interactions with Asp800A, Arg841A, and Leu844A that were seen for Pep were still observed, while new ones were formed with Leu718A and Gly719A. Hydrogen bonds with Lys745A and Asp800A were likewise maintained, while new H-bonds were seen with Met793A, Gly796A, Asp855A, and Asn996A. Mut2 (Figure 6c and Supplementary Information Table S6c) displayed eleven hydrophobic interactions and ten hydrogen bonds as well as a salt bridge with Lys745A. New hydrophobic interactions formed compared to Pep included those with Leu718A, Gly719A, Met793A, Glu804A, Thr854A, Asp855A, and Leu1001A. New hydrogen bonds included those with Leu718A, Ser720A, Met793A, Asn842A, Thr854A, Asp855A, and Asn996A. Mut3, as seen in Figure 6d and Supplementary Information Table S6d exhibited only two hydrophobic interactions and five hydrogen bonds while for Mut4, six hydrophobic interactions and four hydrogen bonds were predicted, along with a salt bridge with Asp800A, as shown in Figure 6e and Supplementary Information Table S6e. Hydrophobic interactions occurred with Val726A, Leu844A, and Leu1001A, while H-bonds were shown with Lys745A, Arg803A, Asp855A, and Lys913A. 


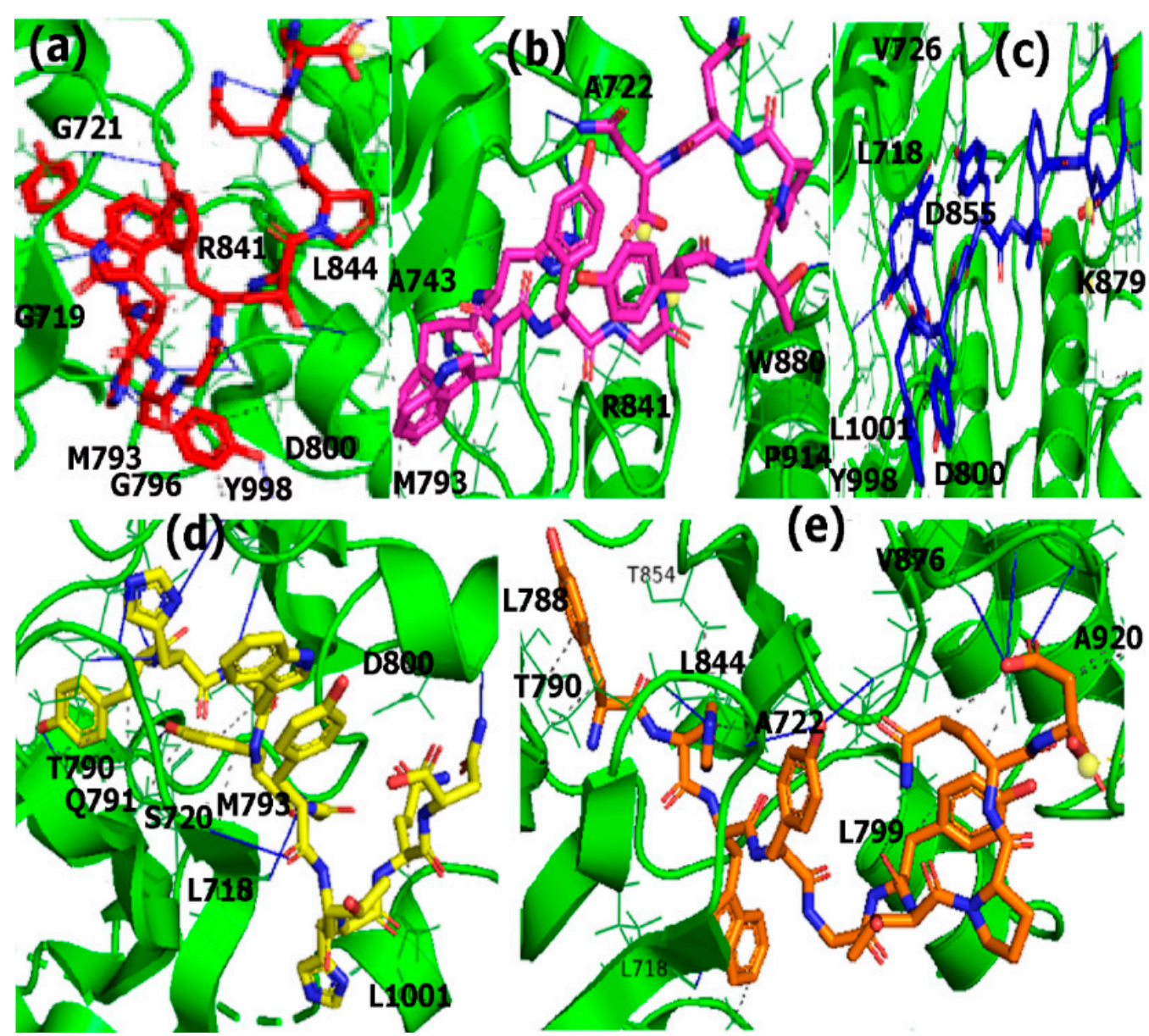

Figure 6. PLIP results for each of the peptides bound to EGFR receptor. (a) Original peptide; (b) Mut1 (c) Mut2 (d) Mut3 (e) Mut4.

PLIP results for polyphenol-peptide conjugates are shown in Figure 7 and Supplementary Information Table S7. For CGA-Pep, as shown in Figure 7a and Supplementary Information Table S7a, six hydrophobic interactions and eight hydrogen bonds occurred, one $\pi$-cation interaction with Arg803A was seen. Some hydrophobic interactions seen for Pep alone were maintained, including those with Leu799A, and Asp800A, while new ones were formed with Ser720A, Ala722A, Val876A, and Pro877A. H-bonds with Asp800A and Arg803A were still seen, while new ones were seen with Gly719A, Ser720A, Gly721A, Cys797A, Arg841A, and Val876A. As indicated in Figure 7b and Supplementary Information Table S7b, eight hydrophobic interactions and seven hydrogen bonds were formed for CSA-Pep. Compared to Pep alone, new hydrophobic interactions were seen with Ser720A, Thr854A, Trp880A, and Lys913A. New hydrogen bonds were observed with Val717A, Leu718A, Ser720A, and Cys797A. GLA-Pep (Figure 7c and Supplementary Information Table S7c) displayed only six hydrophobic interactions and six hydrogen bonds, in addition to one $\pi$-cation interaction with Arg841A. The RMAPep conjugate (Figure $7 \mathrm{~d}$ and Supplementary Information Table S7d) however showed increased interactions comparatively (nine hydrophobic) and ten hydrogen bonds. 


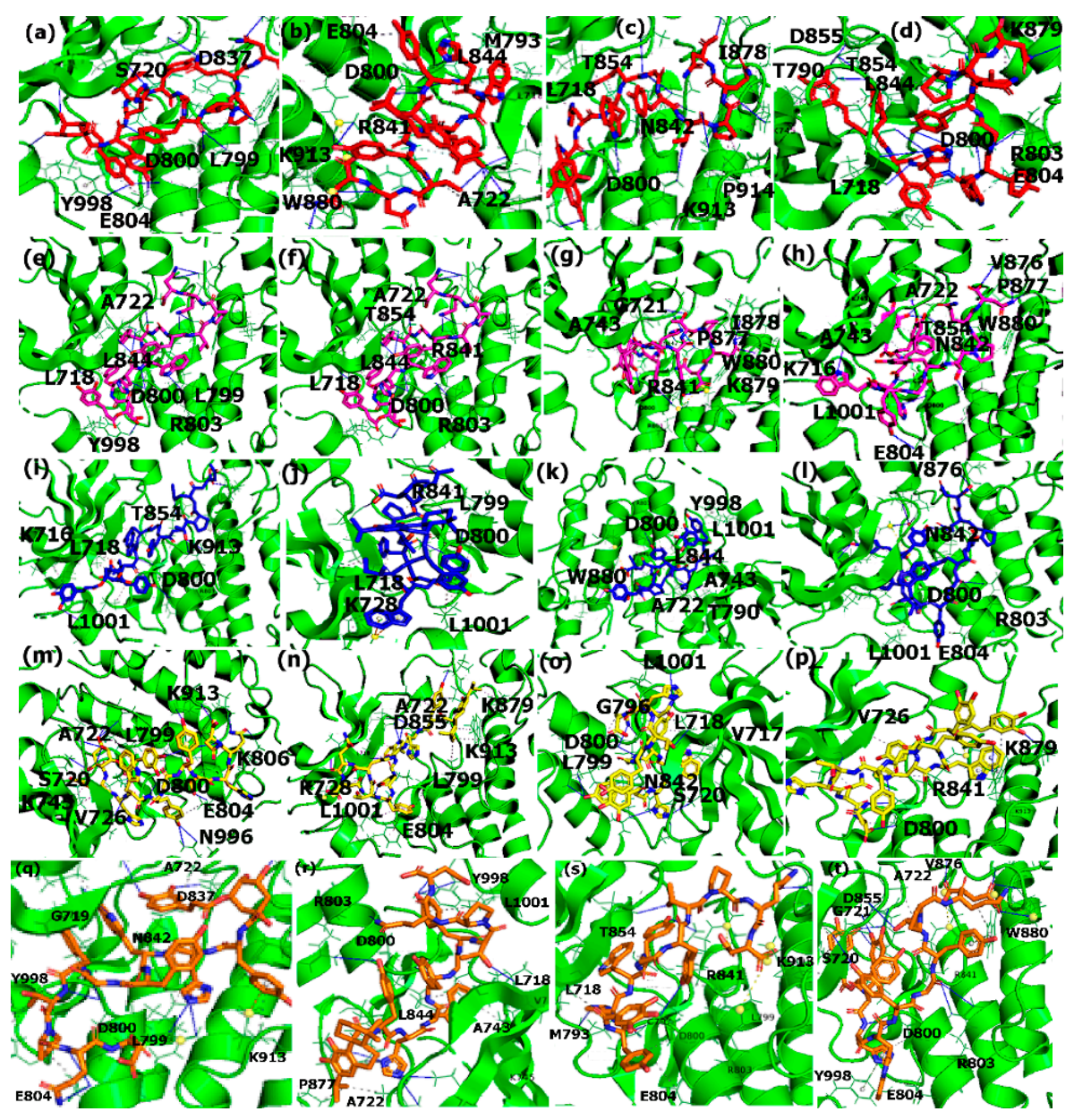

Figure 7. PLIP results for polyphenol peptide conjugates bound to EGFR (a) CGAPep (b) CSAPep (c) GLAPep (d) RMAPep (e) CGAMut1; (f) CSAMut1; (g) GLAMut1; (h) RMAMut1; (i) CGAMut2 (j) CSAMut2; (k) GLAMut2; (1) RMAMut2; (m) CGAMut3; (n) CSAMut3; (o) GLAMut3; (p) RMAMut3; (q) CGAMut4; (r) CSAMut4; (s) GLAMut4; (t) RMAMut4.

CGAMut1, as shown in Figure 7e and Supplementary Information Table S7e, displayed hydrophobic interactions with Ser720A, Val726A, Asp855A, Trp880A, and Lys913A, while new hydrogen bonds were seen with Leu718A, Ser720A, Ala722A, Gly724A, Arg803A, Arg841A, Lys913A, and Asp916A. No interactions observed for Mut1 alone were maintained, indicating a change of position within the EGFR binding site. The CSAMut1 conjugate displayed increased interactions (fifteen hydrophobic interactions and ten hydrogen bonds) as shown in Figure $7 \mathrm{f}$ and Supplementary Information Table S7f. Compared to Mut1 alone, several new hydrophobic interactions and H-bonds were seen. GLA-Mut1 showed lower interactions (eight hydrophobic interactions, nine hydrogen bonds). However, two $\pi$-stacking interactions with Trp880A, and one $\pi$-cation interaction with Arg841A were observed, as shown in Figure $7 \mathrm{~g}$ and Supplementary Information Table S7g. New hydrophobic interactions included those with Val726A, Thr854A, Trp880A, and Pro914A, while new hydrogen bonds occurred with Ser720A and Thr854. RMAMut 1 in comparison displayed thirteen hydrophobic interactions and ten hydrogen bonds, in addition to one $\pi$-cation interaction and one salt bridge with Lys879A as seen in Figure $7 \mathrm{~h}$ and Supplementary Information Table S7h. Compared to Mut1 alone, new hydrophobic interactions occurred with Gly721A, Ala722A, Phe723A, Val726A, Leu799A, Thr854A, Lys879A, and Ala920A, while new hydrogen bonds were observed with Leu718A, Ser720A, Gly721A, Cys797A, Lys879A, Gly911A, Lys913A, and Ala920A.

The CGAMut2 conjugate showed only seven hydrophobic interactions, nine hydrogen bonds, and one salt bridge, as shown in Figure 7i and Supplementary Information Table S7i. 
Compared to Mut2 alone, new hydrophobic interactions were formed with Val717A and Asp800A, while new H-bonds were formed with Val717A, Ala722A, Arg841A, and Lys913A. CSAMut2 comparatively displayed ten hydrophobic interactions and four hydrogen bonds, in addition to one salt bridge with Arg841A, as seen in Figure 7j and Supplementary Information Table S7j. New hydrophobic interactions were seen with Ser720A, Ala722A, Leu799A, Asp800A, and Trp880A. The GLAMut2 conjugate, on the other hand, showed eleven hydrophobic interactions and six hydrogen bonds, as shown in Figure 7k and Supplementary Information Table S7k. Several new hydrophobic interactions were seen, while new H-bonding occurred with Tyr801A, Lys846A, Gln849A, and Met987A. The large change in interactions observed following conjugation of Mut2 with gallate indicates a change of position of the conjugate within the EGFR binding site. RMAMut2 showed only six hydrophobic interactions and eight hydrogen bonds, in addition to two salt bridges with Arg803A and Lys879A, shown in Figure 71 and Supplementary Information Table S71. Compared to Mut2 alone, new hydrophobic interactions were observed with Ser720A, Ala722A, Arg803A, and Trp880A, while new H-bonds formed with Ala722A, Asp800A, Arg841A, Val876A, Lys879A, Gly917A, and Ala920A.

CGAMut3 exhibited seven hydrophobic interactions and ten hydrogen bonds were observed, as well as a salt bridge with Arg841A, indicated in Figure 7m and Supplementary Information Table S7m. Compared to Mut3 alone, new hydrophobic interactions were observed with Leu718A, Gly719A, Ser720A, Leu799A, Asp800A, and Asn996A. New Hbonds were seen with Ser710A, Ala722A, Lys745A, Asp800A, and Asn996A. As seen in for CSAMut3 (Figure 7n and Supplementary Information Table S7n), ten hydrophobic interactions, eight hydrogen bonds, and one $\pi$-stacking interaction were seen. New hydrophobic interactions included those with Ala722A, Val726A, Leu844A, Thr854A, Val876A, Ile878A, and Trp880A, while new H-bonds included those with Lys745A, Arg841A, Val876A, Lys879A, Ile918A, and Ala920A. The GLAMut3 conjugate, as indicated in Figure 7o and Supplementary Information Table S7o, showed nine hydrophobic interactions and eight hydrogen bonds. New hydrophobic interactions included those with Leu718A, Val726A, Asp800A, Leu844A, and Trp880A, while new hydrogen bonds were seen with Leu718A, Asp800A, and Arg841A. RMAMut3 (Figure 7p and Supplementary Information Table S7p) showed seven hydrophobic interactions, including new ones with Leu718A, Gly719A, Leu799A, Asp800A, Lys879A, and Trp880A. Eight H-bonds, including new ones with Val717A, Gly719A, Ser720A, and Val876A, were formed.

CGAMut4, as shown in Figure 7q and Supplementary Information Table S7q, seven hydrophobic interactions, thirteen hydrogen bonds. One salt bridge with Lys745A was observed. Compared to Mut4 alone, new hydrophobic interactions occurred with Lys716A, Leu718A, and Gly719A, while new hydrogen bonding occurred with Val717A, Leu718A, Gly719A, Ser720A, Gly721A, Lys728A, Asp800A, Ala1000A, and Asp1003A. The CSAMut4 conjugate showed shown in Figure 7r and Supplementary Information Table S7r, exhibited eight hydrophobic interactions and twelve hydrogen bonds. New hydrophobic interactions were seen with Gly719A, Phe723A, Ala743A, Thr854A, Glu866A, and Pro877A, while new H-bonds were observed with Ala722A, Phe723A, Arg748A, Arg841A, Thr854A, Gly863A, Val876A, and Arg889A. GLAMut4, on the other hand. displayed only five hydrophobic interactions, but seventeen hydrogen bonds, and one salt bridge as seen in Figure 7s and Supplementary Information Table S7s. A new hydrophobic interaction was seen with Phe723A, while new H-bonds included those with Gly719A, Ser720A, Gly721A, Ala722A, Phe723A, Gly724A, Asp800A, Glu804A, Asn842A, and Asn996A. RMAMut 4, as shown in Figure $7 \mathrm{t}$ and Supplementary Information Table S7t, exhibited nine hydrophobic interactions, including those with Leu718A, Ala743A, Asp800A, Arg841A, and Tyr998A. Fourteen hydrogen bonds were observed, including new ones with Gly719A, Ser720A, Gly721A, Phe723A, Val726A, Gln791A, Met793A, Cys797A, Asp800A, Glu804A, and Asn996A. These results once again indicated that conjugates displayed overall larger interactions and there were changes observed as far as the binding region was concerned. 


\subsection{Molecular Dynamics Studies}

\subsubsection{Analysis of RMSDs and RMSFs for Ligand-Receptor Complexes}

To further elucidate the interactions and examine the stability of the ligands with the receptors, we carried out 100 ns molecular dynamics (MD) simulations. While we conducted MD simulations with all peptides, for the polyphenol-peptide conjugates, only those conjugates that showed significant binding interactions were selected. These include RMAPep, RMAMut1, GLAMut1, GLAMut2, CGAMut4, and CSAMut4.

Figure $8 \mathrm{a}, \mathrm{d}, \mathrm{g}$ shows a comparison of RMSDs of ligand bound receptors of each of the peptides and conjugates with the three receptors. The RMSDs are representative of the last 25 ns of 100 ns runs. The RMSDs of the entire runs are shown in Supplementary Information Figure S1. As shown in the figures, among the unconjugated peptides, Pep and Mut4 showed the lowest RMSDs, followed closely by Mut2 and Mut3 $(<1.0 \mathrm{~nm})$ in the case of ER- $\alpha$. However, Mut1 showed a relatively high RMSD (2.4 nm). In comparison, for PPAR- $\alpha$ the lowest RMSD $(0.3 \mathrm{~nm})$ was observed for Mut2, followed by Pep, Mut1, and Mut4. Mut3 had the highest $(0.82 \mathrm{~nm})$ RMSD in the case of PPAR- $\alpha$. In the case of EGFR, Mut3 was lowest $(0.6 \mathrm{~nm})$ followed closely by Pep, Mut1, and Mut4. The highest RMSD $(0.96 \mathrm{~nm})$ was found to be for Mut2. Thus, in each case the most stable receptor-peptide combination was dependent upon the peptide sequence of the ligand. Furthermore, other than Mut1 (in the case of ER- $\alpha$ ), all of the peptides showed fairly stable binding interactions.
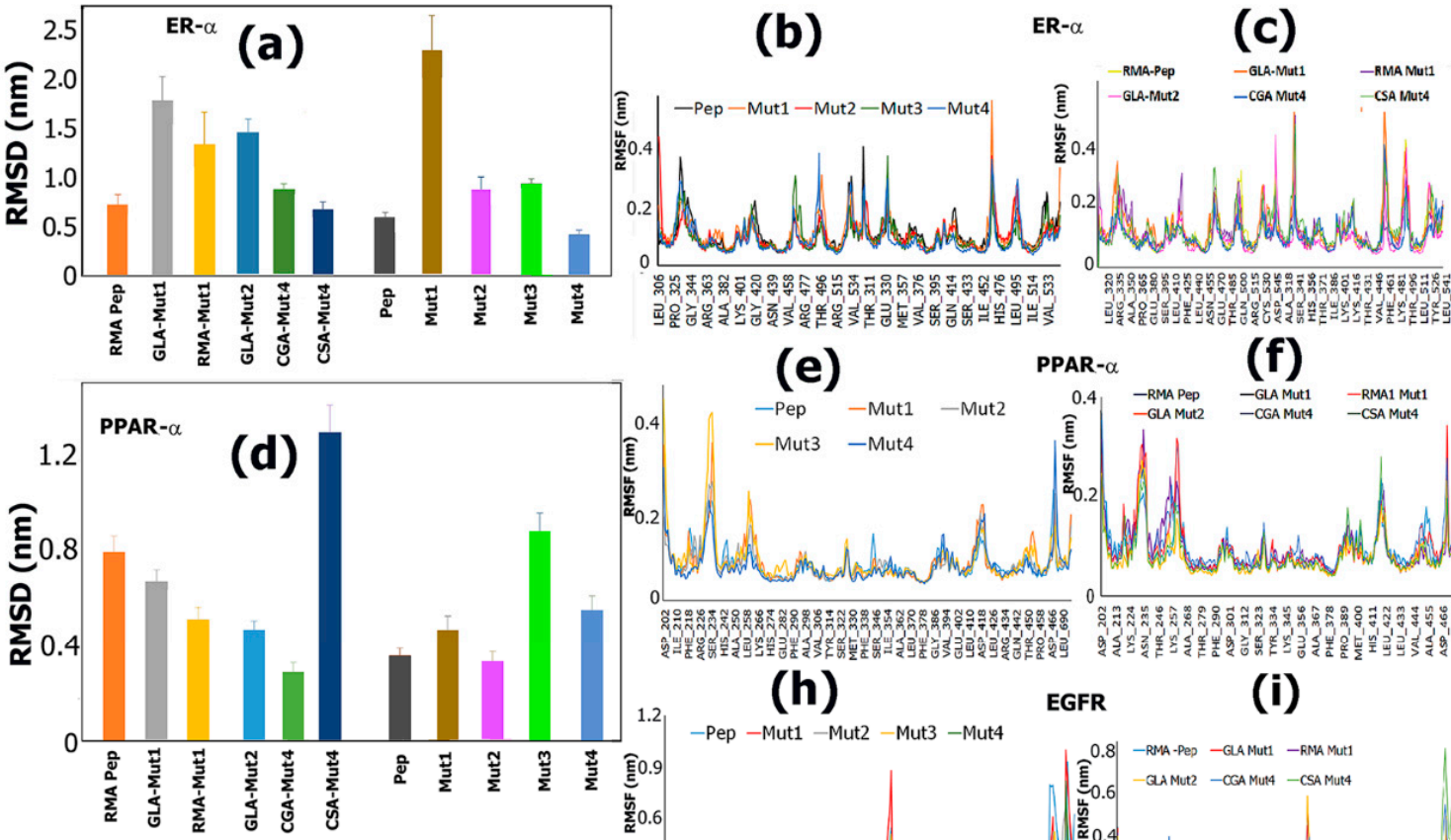

PAR- $\alpha$

(f)

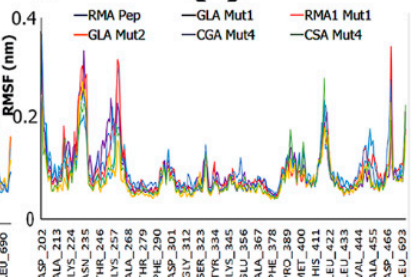

(h)

EGFR

(i)
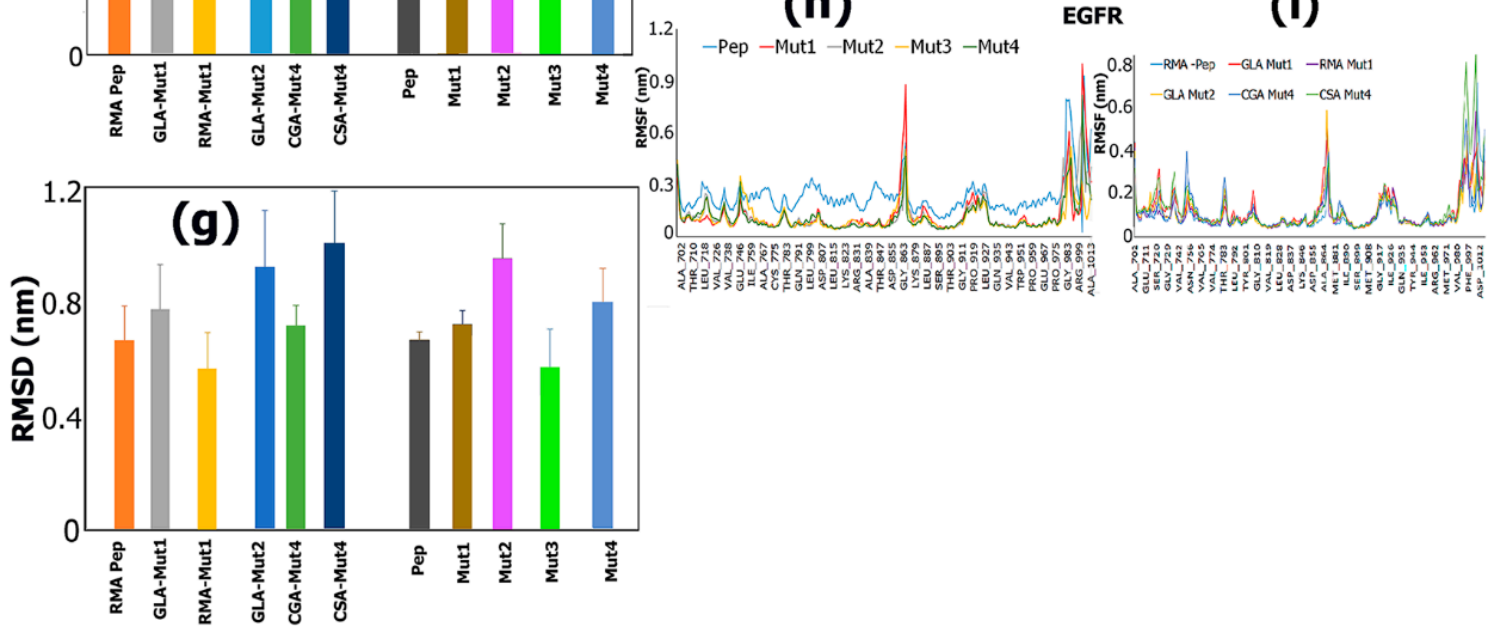

Figure 8. Comparison of RMSDs (72 ns-100 ns). (a) ER $\alpha$; (d) PPAR $\alpha$; (g) EGFR. RMSFs for the entire $100 \mathrm{~ns}$ runs are shown for each of the receptors with (b) and (c) corresponding to ER $\alpha$; $(\mathbf{e}, \mathbf{f})$ corresponding to PPAR $\alpha ;(\mathbf{h}, \mathbf{i})$ corresponding to EGFR. 
Amongst the polyphenol-peptide conjugates, RMA-Pep was found to have lower RMSD values $(\sim 0.7 \mathrm{~nm})$ in the case of all the three receptors. GLAMut1, was found to have the highest RMSD for ER- $\alpha$, while CSAMut4 was found to have the highest RMSD for both PPAR- $\alpha$ and EGFR in this time frame, indicating that toward the end of the simulations those ligands are relatively less tightly-bound to the respective receptors. Interestingly, CSAMut4 was found to have the lowest RMSD for ER- $\alpha$, indicating that it was the most stable conjugate with ER- $\alpha$. CGAMut4 showed the lowest RMSD with PPAR- $\alpha$, while RMAMut1 showed the lowest RMSD with EGFR indicating the formation of highly stable complexes [65].

The RMSF graphs for ER- $\alpha$ (Figure $8 b, c)$ show the fluctuations of the receptor upon binding with the various ligands. In the case of the peptides (Figure $8 b$ ), while several regions showed fluctuations, Mut3 showed highest fluctuations with Leu466 and Asp332, while Mut4 showed the highest fluctuations with Gly494 and Leu497. Mut1 showed the highest fluctuation with Lys467 and Pep showed highest fluctuations with Asp322 and Ala546, indicating the strong involvement of those residues in binding interactions. Comparatively, more fluctuations were observed for the polyphenol-peptide conjugates (Figure 8c). GLAMut2, GLAMut1, RMAMut1, showed the highest fluctuations with Ser305, Tyr331; Lys467 and Glu419 respectively.

To further probe these results, we examined the trajectory snap shots of the peptide and conjugate-bound ER- $\alpha$ at various time points throughout the simulation (Supplementary Information Figure S2). In general, estrogen receptors are composed of two domains, made up mostly of $\alpha$-helices. It's native ligand, estradiol, binds within a largely hydrophobic pocket of ER $\alpha$ through H-bonds with Glu353, Arg394, Phe404, and His524 residues in the ligand binding domain of the receptor [66].

Pep initially has a strong association with the receptor in the binding pocket region encompassing multiple helices. The ligand binding domain of ER- $\alpha$ consists of a triplelayered anti-parallel $\alpha$-helical sandwich and a small anti-parallel $\beta$-sheet at one end $[67,68]$. Through the course of the simulation, the ligand (depicted in red) changes conformation and by the end of the simulation it is attached to the central layer comprised of $\mathrm{H} 3 / \mathrm{H} 6 / \mathrm{H} 9 / \alpha-$ helices in the region. A slight conformation change is also observed in the receptor where the $\mathrm{H} 4$ helix at the bottom changes to a bent shape in order to best fit the ligand. For Mut1, the ligand is seen within the estrogen receptor binding throughout the first $50 \mathrm{~ns}$ of the simulation, though it begins to migrate within the receptor and reaches all the way to the top of H6 helix. By 100 ns, the Mut1 peptide has migrated out of the receptor completely, explaining the large RMSD at the end of the simulation. Mut 2 undergoes very minor conformational changes and remains associated with the receptor primarily through Helix 6 and Helix 3. Thus, Mut2 appears stable within the binding site. Mut 3 undergoes conformational changes during the simulation, where it appears to move slightly more toward the Helix 6 region at the 75 ns time point and remains stable at that position for the rest of the simulation. Several residues remain associated with the receptor throughout; it appears that both $\mathrm{N}$-terminal and $\mathrm{C}$-terminal regions of the Mut3 interact with the receptor at the end of the simulation. Mut4 appears to migrate further inward throughout the $100 \mathrm{~ns}$, indicating that further interactions are forming between the peptide and the receptor during the simulation. At 100 ns, Mut 4 is nestled tightly within the receptor.

The trajectories of the polyphenol-peptide conjugates with ER- $\alpha$ are shown in Supplementary Information Figure S3. RMAPep moves slightly in relation to ER- $\alpha$ throughout the simulation and shows conformational changes, appearing to spread more widely to associate with ER- $\alpha$ by the end of the simulation, at which point it is attached to $\mathrm{H} 3, \mathrm{H} 6$ and H9 helices. GLAMut1, on the other hand, appears to move away from the binding site and moves toward the $\mathrm{H} 9$ and $\mathrm{H} 10$ helices but remains attached to the receptor throughout the simulation. RMAMut1 also changes position slightly in the first $25 \mathrm{~ns}$, after which minimal change occurs, indicating stability of RMAMut1 in complex with ER. GLAMut2 appears to jump around within the binding pocket at different positions but remains at the same position in the region underneath the binding pocket after 75 ns. CGAMut 4 remains in the 
same position throughout the simulation, appearing elongated and indicating favorable interactions with $\mathrm{ER} \alpha$. CSAMut4 likewise remains in the same position, but starts in a folded conformation, and after 25 ns appears more elongated. Minimal conformation change occurs between $75 \mathrm{~ns}$ and $100 \mathrm{~ns}$, implying stability in complex with ER- $\alpha$.

RMSF graphs for PPAR- $\alpha$ with the peptides (Figure 8e) showed the highest fluctuations with Arg226, Ala250 and Asp418 and Asp466, with Mut3 resulting in the highest fluctuation with Arg226. On the other hand, the peptide-polyphenol conjugates (Figure 8f) showed major fluctuations, particularly residues Lys232, Lys252, Gly262, Leu392, Asp418, Asp453 and Ala685. The highest fluctuations were seen for RMA-Mut1, CSA-Mut4 and GLA-Mut1 indicating higher interactions.

To further probe these results, we analyzed the corresponding MD trajectories. Supplementary Information Figure S4 shows the MD trajectory images of each peptide with PPAR- $\alpha$ at 0 ns, 25 ns, 50 ns, 75 ns, and 100 ns. Each of the peptides bind to the ligand binding domain within the alpha-helices [69]. Pep shows very little change in conformation throughout the simulation whereas parts of Mut1 seem to rotate slightly within the binding pocket. Mut 2 shows a significant change between its position at 0 ns and 25 ns but remains very stable from $25 \mathrm{~ns}$ till the end of the simulation. Mut3 seems to gradually migrate up the binding pocket throughout the $100 \mathrm{~ns}$ while also increasing in compactness by slightly folding in on itself. All peptides are shown to remain fully attached to the PPAR- $\alpha$ receptor for the entire simulation, while Mut1 appears the least strongly bound and Mut2 appears the most tightly attached.

We then examined the trajectories of the polyphenol-peptide conjugates with PPAR- $\alpha$ (Supplementary Information Figure S5). Like the peptides, most of the polyphenol-peptide conjugates appear to initially bind within the binding pocket of the PPAR- $\alpha$ receptor (except CSAMut4 and CGAMut4). RMAPep appears the most compact within the receptor compared to all other compounds. GLAMut1 shows more movement within the receptor binding pocket, especially in the first $25 \mathrm{~ns}$, and it remains mostly unfolded throughout the simulation. Furthermore, it appears to move away from the helical region into the loop region after $50 \mathrm{~ns}$. RMAMut1 also shows a slight initial change in position followed by high stability within the binding pocket and keeps a relatively less compact, more open conformation. GLAMut2 also shows a slight initial change in position followed by high stability within the binding pocket with a relatively less compact conformation. The binding for GLAMut1, RMAMut1, and GLAMut2 look very similar in terms of ligand position within the binding pocket and ligand compactness. CGAMut4 and CSAMut4 are relatively loosely attached to the PPAR- $\alpha$ binding pocket and are attached on the peripheral alpha helical regions and loops. Additionally, the ligands remain partly outside of the binding pocket. However, CGAMut4 remains in the same position for the entirety of the simulation whereas CSAMut4 shows significant movement. CGAMut4 and CSAMut4 also show binding on opposite sides of the PPAR- $\alpha$ receptor. Based on the trajectory images, CSAMut4 appears the least attached and least stable while RMAPep appears to remain the most well attached to the receptor.

The EGFR model structure selected is that of the EGFR kinase domain, which has a bilobate structure, with the N-terminal group consisting of mostly beta-strands and one alpha-helix while the C-terminal lobe constitutes of primarily $\alpha$-helical structures. The cleft to which several ligands and ATP analogues are found to bind is found between the two lobes [70]. The catalytic domain is surrounded by the cleft and consists of the glycinerich nucleotide phosphate-binding loop (Gly695-Gly700), while the C-lobe contributes the DFG motif (Asp831-Gly833), the catalytic loop (Arg812-Asn818), and the A-loop (Asp831Val852) [71]. The RMSF graphs for EGFR are shown in Figure 8h,i corresponding to the peptide-bound receptor and the polyphenol-peptide bound receptor, respectively. For the peptide-bound receptors, major fluctuations are observed with Lys867 along with Ser921, Lys929 and Met987 with the highest fluctuations being seen for Mut1 and Pep. For the conjugates, fluctuations were observed at the same residues as the peptide, along with those seen at Ala722, Val738, Arg748 and Ser784. The later residues showed less intense 
fluctuations comparatively, though the values were higher for the conjugates compared to the unconjugated peptides. In previous work, it has been observed that the residues located in the loop or helix parts of receptors show higher RMSF fluctuations particularly in the $N$ and C-terminal regions [72]. The same was also observed in the case of the peptides and the polyphenol conjugate interactions with EGFR.

To further probe these results, we analyzed the corresponding trajectories (Supplementary Information Figure S6). Pep appears to bind to the hinge region. For the first $50 \mathrm{~ns}$ of the simulation, the ligand appears to undergo conformation changes within the cleft; however, after 75 ns, minimal changes were observed, indicating that the ligand was tightly bound to the pocket by the end of the simulation. Mut1 also attached to the hinge region; however, at $25 \mathrm{~ns}$, the ligand appeared to change conformation, fold up and move slightly away from the alpha-helix adjacent to the C-lobe. The ligand remains stable for the entirety of the simulation at this position after $25 \mathrm{~ns}$. The Mut2 peptide appeared to bind in the same region as well; however, during the course of the simulation, it appeared to fold up, between $50 \mathrm{~ns}$ and $75 \mathrm{~ns}$ but, by the end of the simulation, it showed binding with the alpha-helix region adjacent to the $\mathrm{C}$-lobe. For the Mut3 peptide, the ligand appeared to make more contacts with the receptor, encompassing the G-loop region, as well as the alpha-helices, and further expanded and reached out to the C-lobe region during the course of the simulation. This also explains the RMSD values, showing the most stable conformation for the Mut3 peptide with EGFR. In the case of Mut4, it also bound within the hinge region and, after $25 \mathrm{~ns}$, its conformation remained stable and no changes were observed, confirming that Mut4 also formed a very stable complex with the receptor.

We next examined the trajectories of the polyphenol-peptide conjugates with EGFR. As shown in Supplementary Information Figure S7, for RMAPep, the ligand bound to the hinge region and encompassed the alpha-helix motif adjacent to the C-lobe. The conformation of the ligand underwent very few changes over the entire simulation process, indicating its stability once attached to the receptor. For GLAMut1, a similar result was observed, where the ligand, once attached to the receptor, underwent very few changes and remained tightly attached. In the case of RMAMut1, the ligand initially attached to the hinge region and encompassed multiple alpha-helices in the C-loop region, however, at $25 \mathrm{~ns}$, the ligand appeared to flip, and the aromatic rings of the RMA moiety appeared to bind to the G-loop region and the peptide component moved toward the alpha-helices of the C-lobe. At the end of the simulation, the ligand appeared to move further inward, attaching more with the alpha-helices on the C-lobe. The trajectory of GLAMut2, however, showed a completely different story. While, initially, it appeared to be below the binding pocket, during the course of the simulation, it appeared to move inward, fold into itself, and then appeared to bind minimally to the loop region. This explains the large RMSD after $75 \mathrm{~ns}$, where the ligand position changes completely as it initially moves away and then moves closer. This indicates that GLAMut2 shows relatively weak binding initially. For CGAMut4, the ligand remained attached to the binding pocket throughout the simulation process, though slight changes occurred in the conformation of the ligand. It is interesting to note however, that in the case of this ligand, after $50 \mathrm{~ns}$, the ligand appeared to more toward the beta-sheet region of the N-lobe rather than the C-lobe, towards which the other ligands appeared to move. For CSAMut4, only part of the ligand binds to the G-rich loop of the cleft region, while a substantial portion of the ligand appears to bind above the cleft region. Furthermore, the ligand appeared to change conformation at the $50 \mathrm{~ns}$ to $75 \mathrm{~ns}$ range and appeared to move toward the $\mathrm{C} \alpha$-helix region, though a major part of the ligand seemed to be away from the receptor. This further confirms that this particular ligand-receptor complex is relatively less stable compared to some of the other ligands, which remain tightly attached throughout the simulation process. 


\subsubsection{Protein-Ligand Interactions}

$\mathrm{ER}-\alpha$

To further elucidate the interactions, the protein-ligand contacts were examined. Figure 9 shows the results obtained for ER- $\alpha$. As shown in Figure 9a, protein ligand (P-L) contacts for Pep with ER- $\alpha$ were dominated by H-bonds and water bridges. Sites of significant H-bonding and water bridges include Glu380B, Ser463B, and Glu523D. A small number of hydrophobic interactions were also seen. A total of 20 contacts were observed on average throughout the course of the simulation (Figure 9b). For Mut1 (Figure 9c) P-L contacts were dominated by H-bonds and water bridges. Glu419D, Ser527D, and Cys417D were sites of significant H-bonding. Some hydrophobic interactions were observed, particularly at Tyr537B. The total number of contacts (Figure 9d) initially started off at an average of 24, which reduced to almost zero by the end of the simulation, which is expected based on the trajectory data that showed that the Mut1 ligand moved away from the receptor by the end of the simulation. For Mut2, the P-L interactions (Figure 9e) were dominated by H-bonds, particularly with Glu380B and Asp426B. Significant hydrophobic interactions occurred at Tyr537B, which was also indicated by PLIP. The total number of contacts throughout the course of the simulation was found to be 15 on average (Figure $9 \mathrm{f}$ ). For Mut3, the P-L contacts (Figure 9g) are mainly H-bonds, with major interaction sites at Leu462B, Val534B, and Asp462D. Sites of hydrophobic interactions include Tyr537B and Phe461B. The total number of contacts was found to be 18 , on average, with higher contacts in the initial stages of the simulation (Figure 9h). In the case of Mut4, P-L interactions (Figure 9i), show mainly H-bonds, with the highest interaction fraction being for Glu380B. Hydrophobic interactions are observed with Trp383B and Tyr537B. These results are also consistent with PLIP studies. The total number of contacts throughout the simulation was found to be 24 on average with higher contacts (30) at the 5 ns time point (Figure 9j). All of the corresponding protein-ligand atom contacts are shown in Supplementary Information Figure S8.

The P-L contact histograms for the polyphenol-peptide conjugates with ER- $\alpha$ are shown in Figure 10. For RMA-Mut1 (Figure 10a), H-bonding, particularly with Tyr537B, Asp538B, and Leu462B is observed. Residues mainly involved in hydrophobic interactions include His373B and Tyr537B. The Tyr537B residue is thus involved in both H-bonding and hydrophobic interactions. The total number of contacts (Figure 10b), on average, was found to be 10, with major dips in the number of contacts in the $20 \mathrm{~ns}$ and $35 \mathrm{~ns}$ region, after which the number of contacts remains relatively steady after $40 \mathrm{~ns}$ at about 10 contacts. P-L contacts for RMAPep with ER- $\alpha$ (Figure 10c) showed extensive H-bonding occurred with Glu423D, Tyr526B, and Glu380B of ER. Hydrophobic interactions were seen with Lys520D, while Glu380B and Glu423D were involved in ionic interactions. Tyr526B and Glu380B were also involved in H-bonding between RMAPep and ER- $\alpha$, in accordance with PLIP results, while Glu423D and Lys520D were also implicated as interacting residues. The total number of contacts was found to be 22 contacts, on average, with the highest number of contacts being 30 at 40 ns (Figure 10d).

The P-L contacts with GLAMut1 are shown in Figure 10e. H-bonding occurred with Lys531B, Ser536B, and Glu542B, while hydrophobic interactions were observed with Tyr526B, Leu539B. Lys531B, and Lys531B and Asn532B were additionally involved in ionic interactions. The total number of contacts, on average, (Figure 10f) was found to be 8 , with the highest being 15 at the 40 ns and 65 ns range, after which the number of contacts remained steady at 8. P-L contacts (Figure 10g) between GLAMut2 and ER- $\alpha$ showed several H-bonding interactions occurred with Ser463B, Leu462B, and Ser464B being prominent. Hydrophobic interactions were seen with Try537B and His373B. The total number of interactions (Figure 10h) through the course of the simulation was found to be 9 on average, with the highest number of contacts (15) at 25 ns. P-L contacts between CGAMut4 and ER $\alpha$ are shown in Figure 10i. Significant $\mathrm{H}$-bonding was observed with Glu423D, Lys531B, Asp374B, and Val534B. Hydrophobic interactions occurred with Tyr537B, Tyr526B, and His377B, while Glu423D and Asp538B were involved in ionic interactions. In 
PLIP results, Glu423D, Lys531B, and Asp374B were involved in H-bonding and Tyr537B and Tyr526B were involved in hydrophobic interactions. The total number of contacts (Figure 10j) was found to be 20 over the course of the entire simulation, which is expected given the RMSD analysis which showed a very stable complex. As shown in the P-L contacts histogram for CSAMut4 (Figure 10k), H-bonding occurred with Glu423D, His516D, Glu523D, and Tyr526B of ER $\alpha$. Hydrophobic interactions occurred with Lys520D and Tyr526B, while Glu423D was involved in ionic interactions. Glu423D, His516D and Tyr526B were also implicated in H-bonding in PLIP results. The total number of contacts throughout the entire course of simulation (Figure 101) was found to be 15. Thus, the protein-ligand contact analysis further confirms the role of $\mathrm{H}$-bonding and hydrophobic interactions involved in forming complexes with each of the ligands. The ligand atom interactions with the receptor residues are shown in Supplementary Information Figure S9, which corroborates with the results obtained above.
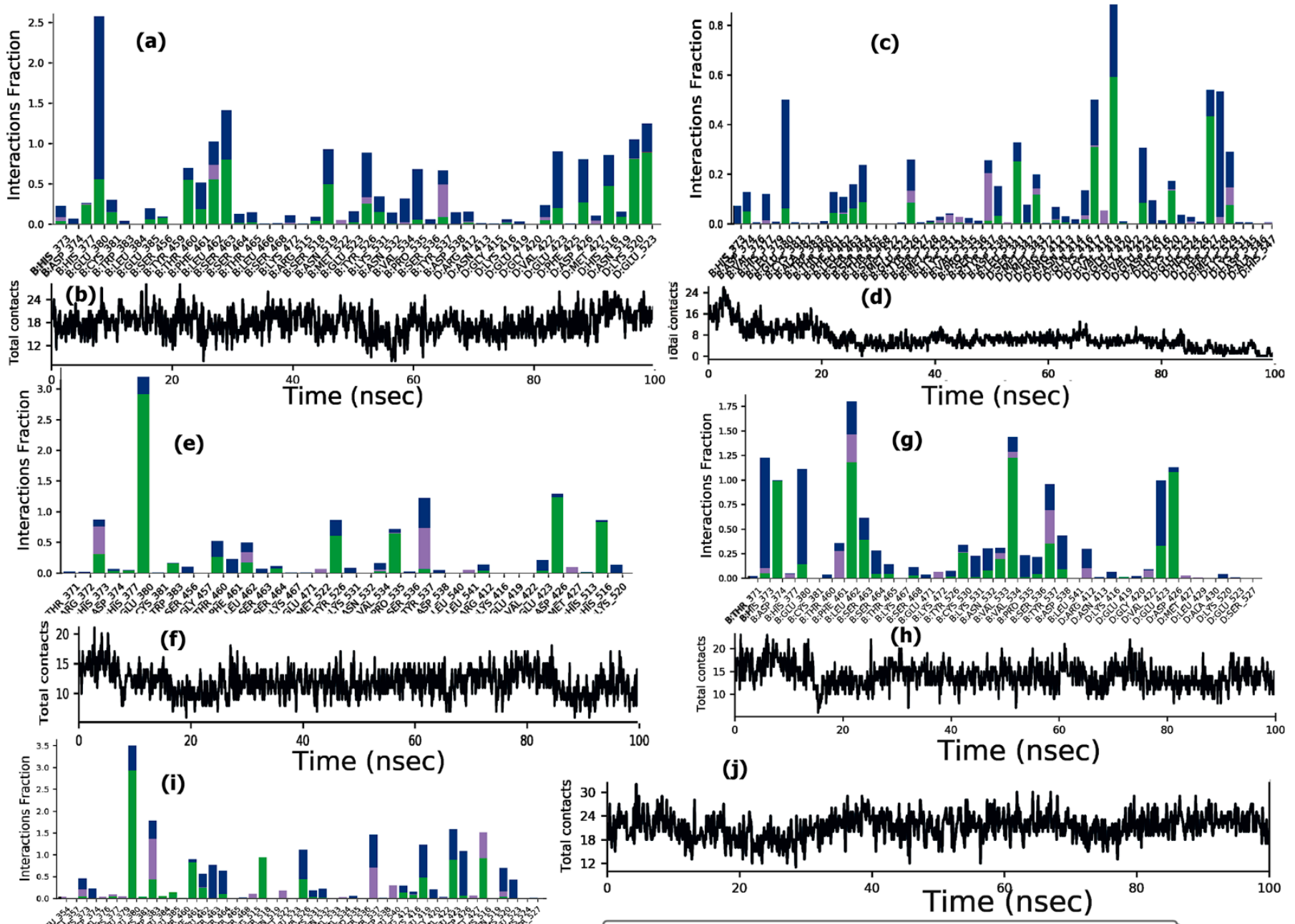

(j)



Figure 9. Protein ligand contacts of ER $\alpha$-ligand complexes (a) with Pep; (b) total number of contacts during the simulation with Pep; (c) with Mut1; (d) total contacts during the simulation with Mut1; (e) with Mut2; (f) total number of contacts during the simulation with Mut2; (g) with Mut3 peptide; (h) total number of contacts during the simulation with Mut3; (i) with Mut4 peptide; (j) total number of contacts during the simulation with Mut4. 

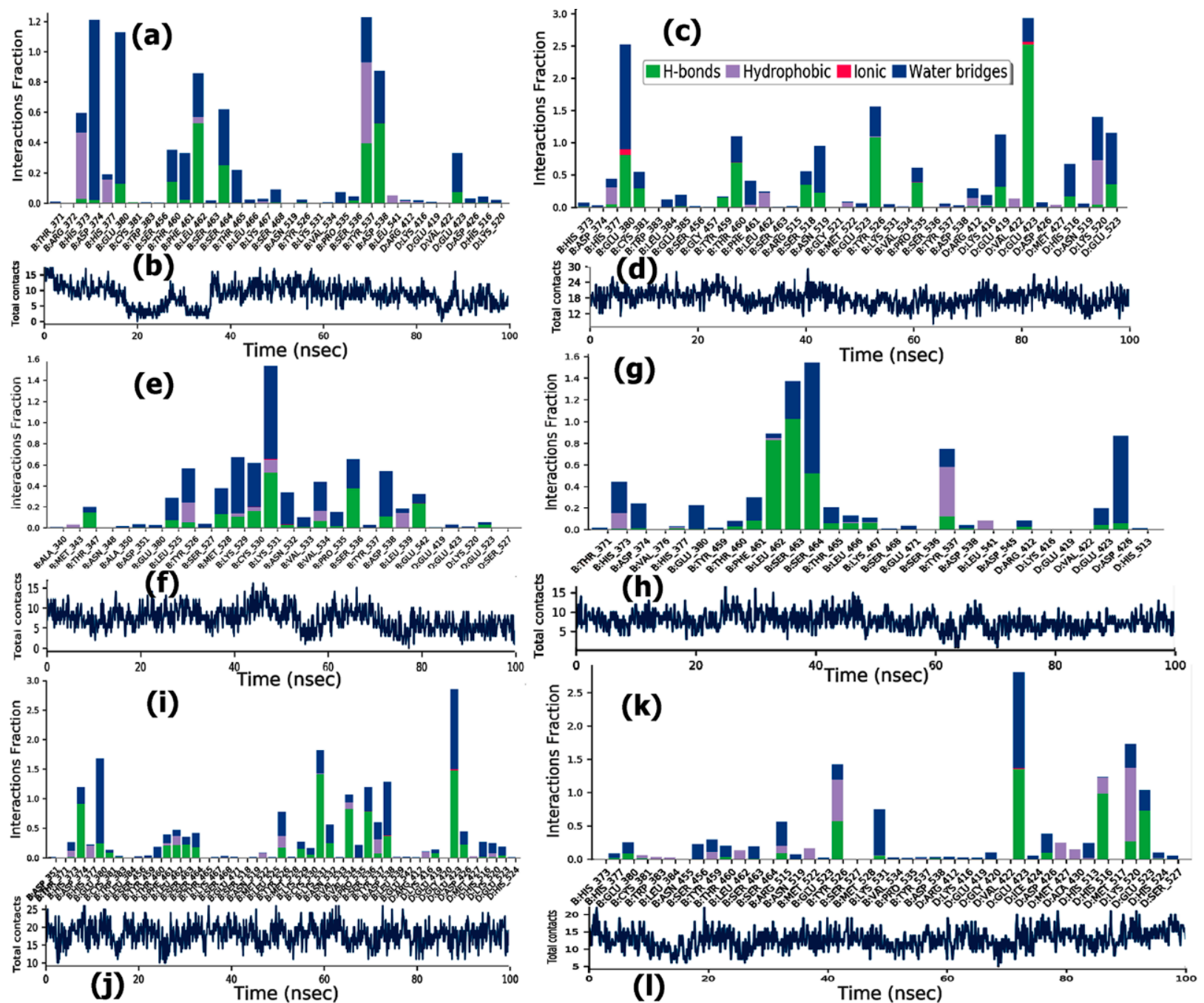

Figure 10. Protein ligand contacts of ER $\alpha$-ligand complexes. (a) with RMAMut1; (b) total number of contacts with RMAMut1; (c) with RMAPep; (d) total contacts with RMAPep; (e) with GLAMut1; (f) total number of contacts with GLAMut1; (g) with GLAMut2 conjugate; (h) total number of contacts with GLAMut2; (i) with CGAMut4; (j) total number of contacts with CGAMut4; (k) with CSA-Mut4; (1) total number of contacts with CSAMut4.

Overall, MD studies of the ER $\alpha$-peptide and polyphenol-peptide conjugate complexes provided valuable insight into the stability of each peptide and polyphenol-peptide construct in complex with the receptor. Of the unconjugated peptides, Mut4 showed the greatest degree of stability with ER- $\alpha$. Of the polyphenol-peptide conjugates, CGAMut 4 and CSAMut4 showed favorable degrees of stability, as did RMAPep, which showed comparable stability compared to Pep. Overall, polyphenols conjugated to the peptides were involved in higher numbers of interactions with the receptor, indicating that conjugation of polyphenols to the targeting peptides could improve ER- $\alpha$ targeting.

PPAR- $\alpha$

We next probed the PPAR- $\alpha$ receptor residues that were involved in interacting with each of the ligands (Figure 11). As seen in the case of the original peptide and mutations $1-4, \mathrm{H}-$ bonding interactions and water-bridges played a major role in the binding interactions. Pep, Mut1, Mut4 and Mut2 showed significantly more H-bonding interactions. Additionally, Pep, Mut2 and Mut3 showed significant hydrophobic interactions. 

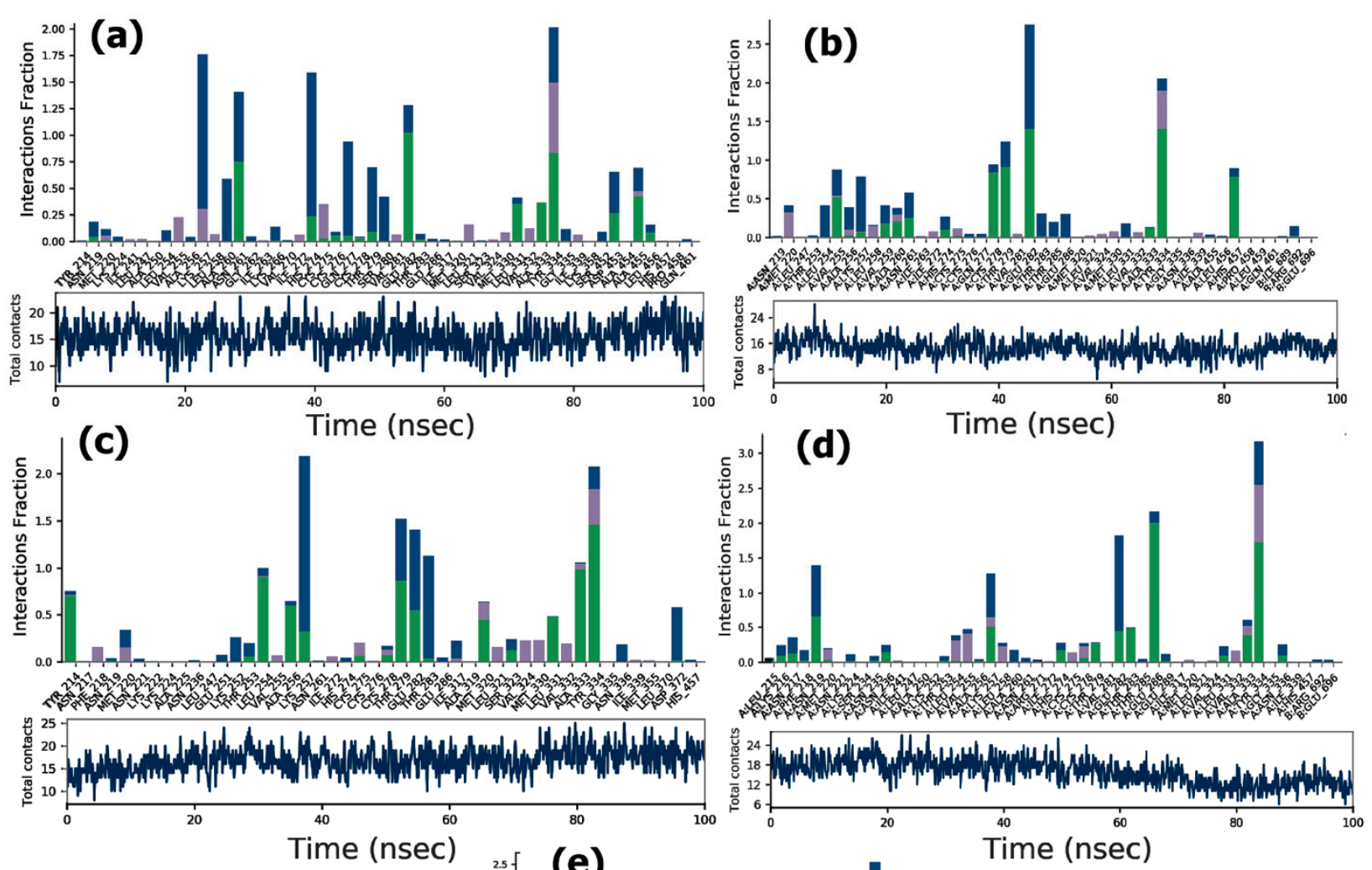

H-bonds Hydrophobic

lonic $\square$ Water bridges

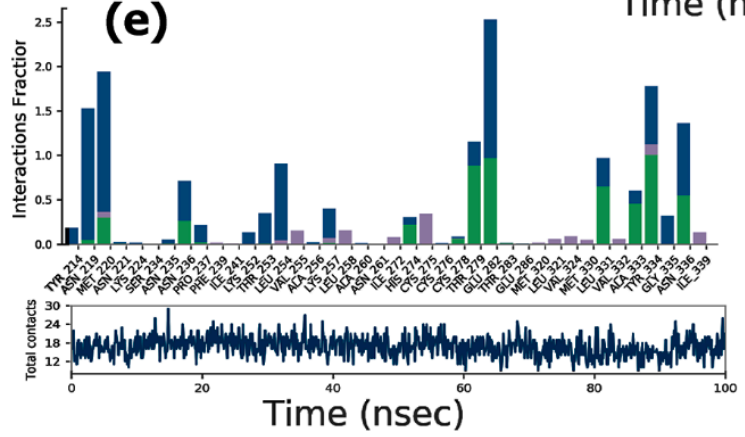

Figure 11. Protein ligand contacts of PPAR $\alpha$-ligand complexes with (a) Pep (top); total number of contacts with Pep (bottom); (b) Mut1 (top); total number of contacts with Mut1 (bottom); (c) Mut2 (top); total number of contacts with Mut2 (bottom); (d) Mut3 (top); total number of contacts with Mut3 (bottom); (e) Mut4 (top); total number of contacts with Mut4 (bottom).

In all five peptides, Tyr 334 showed significant $\mathrm{H}$-bonding and hydrophobic interactions. For Pep (Figure 11a), Asn 261, Glu 282, and Tyr 334 showed the most H-bonding. The total protein-ligand contacts steadily fluctuated around 15 for the entire simulation. For Mut1 (Figure 11b), Glu 282 showed significant H-bonding. The total protein-ligand contacts also steadily fluctuated around 15 for the entire simulation. For Mut2 (Figure 11c), Thr 279 and Glu 282 also showed significant H-bonding. The total P-L contacts showed a gradual increase from 15 to 20 throughout the simulation. For Mut3 (Figure 11d), similar residues showed H-bonding, but Mut3 appeared to have the lowest number of P-L contacts of all the peptides. The total P-L contacts showed a gradual decrease from 18 to 12 throughout the simulation. For Mut4 (Figure 11e), Tyr334 showed significantly less H-bonding and hydrophobic interactions, indicating that the Tyr334 residue was less able to interact with the receptor due to the mutations of Mut4. The total protein-ligand contacts fluctuated around 18 for the simulation. In previous studies, Thr279 has been implicated in binding of ligands to PPAR- $\alpha$ [73], as has Tyr334. This indicates that that some of the conjugates also interact in the same region. The interactions showing the ligand-atom contacts for each of the peptide ligands with PPAR- $\alpha$ are shown in Supplementary Information Figure S10. 
For the polyphenol-peptide conjugates, results for the protein-ligand contacts obtained are shown in Figure 12. For RMAPep (Figure 12a), Glu286 played a major role in H-bonding followed by Tyr334, which was also implicated in hydrophobic interactions in addition to several other residues. The total P-L contacts increased in the first $30 \mathrm{~ns}$ of the simulation and then steadily fluctuated around 20 contacts. For RMAMut1 (Figure 12b), Glu282 played a major role in both H-bonding and water bridges in addition to Tyr 334, His 457 and Lys 257 which also showed hydrophobic interactions. The total protein-ligand contacts fluctuated around 18 for the first $60 \mathrm{~ns}$ and then dropped to around 15 for the remainder of the simulation. For GLAMut1 (Figure 12c), Tyr334 showed the highest interaction fraction involved in H-bonding, hydrophobic interactions, and water bridges. The total protein-ligand contacts varied throughout the simulation, showing an overall decrease to about 18 contacts by the end of the simulation. For GLAMut2 (Figure 12d), more $\mathrm{H}$-bonding was seen than any other conjugate, with Glu286 playing the largest role in H-bonding. The total protein-ligand contacts remained very steady throughout the simulation, fluctuating around 18 contacts. For CGAMut4 (Figure 12e), Glu282 played a large role in water bridges, but many residues seemed to play an important role in interacting with the receptor. The total protein-ligand contacts showed a gradual increase throughout the simulation, ending with about 24 contacts. Finally, for CSAMut4 (Figure 12f), Arg226 played a major role in H-bonding, hydrophobic interactions, and water bridges. The total protein-ligand contacts showed an initial drop from about 10 to 5 contacts in the first $5 \mathrm{~ns}$ of the simulation, followed by a slight increase to about 7 contacts by the end of the simulation. Overall, the different polyphenol-peptide conjugates showed very different protein-ligand contacts with highest interactions being observed for CGAMut4; RMAMut1 and GLAMut1. Tyr 334 interactions were found to be common in all cases, indicating a combination of hydrophobic and $\mathrm{H}$-bonding interactions which stabilized these complexes. The individual ligand-atom interactions for each of these complexes are shown in Supplementary Information Figure S11, which corroborates with the histogram results obtained for the Protein-Ligand contacts. Overall, for all polyphenol-peptide conjugates, $\mathrm{H}$-bonding interactions, hydrophobic interactions and water-bridges played a major role in binding interactions.


H-bonds Hydrophobic $\square$ lonic $\square$ Water bridges

Figure 12. Protein ligand contacts of PPAR $\alpha$-ligand complexes. (a) with RMAPep conjugate (top); total contacts during the simulation with RMAPep conjugate (bottom); (b) with RMAMut1 conjugate (top); total contacts with RMAMut1 conjugate (bottom); (c) with GLAMut1 conjugate (top); total contacts with GLAMut1 (bottom); (d) with GLAMut2 conjugate (top); total contacts with GLAMut2 (bottom); (e) with CGAMut4 conjugate (top); total contacts with CGAMut4 (bottom); (f) with CSAMut4 conjugate (top); total contacts with CSAMut4 (bottom). 


\section{EGFR}

For EGFR P-L contacts, for Pep and Mutations 1-4 (Figure 13), H-bonding interactions and salt-bridges played a major role in the binding interactions. In particular, Asp800 was found to have a very high interaction fraction in all cases. While fewer hydrophobic interactions were seen in the case of Pep (Figure 13a), relatively higher hydrophobic interactions were observed for Mut1 (Figure 13b) as expected given that the N-terminal Y is mutated to F. These interactions are seen with Phe997, Tyr998 and Leu1001 in addition to Leu844, Leu718, and Lys716, which are also involved in H-bonding. Interestingly, a new ionic interaction is observed with Lys745. For Pep, the total number of protein-ligand contacts was found to be in the average range of 16-18, while for Mut1, the average contacts for the entire simulation increased to 21 .


Figure 13. (a) Protein ligand contacts of EGFR-ligand complexes. (a) with Pep (top); total contacts with Pep (bottom); (b) with Mut1 (top); total contacts with Mut1 (bottom); (c) with Mut2 (top); total contacts with Mut2 (bottom); (d) with Mut3 (top); total contacts with Mut3 (bottom); (e) with Mut4 (top); total contacts with Mut4 (bottom). 
For Mut2 (Figure 13c), the number of contacts throughout the simulation process was lower than for Mut1 and was found to be 17. Overall, H-bond interactions were the dominant interactions, while hydrophobic interactions also played a role. No ionic interactions were observed, given that the His at position 2 of the original peptide was mutated to Ile. For Mut3 (Figure 13d), a very high interaction fraction was observed for Asp800 (4.0, highest among all the peptides). Other residues involved also showed higher interactions (between 0.7-1.0) compared to Mut1, 2 and Pep. In addition to H-bonding and water-bridges, a few hydrophobic interactions were also observed. Additionally, on average, about 22 contacts were observed throughout the simulation process. For Mut 4 (Figure 13e), once again Asp800 showed a high interaction fraction due to H-bonding and water-bridges, though it was lower than that observed for Mut3. Overall, relatively lesser residues were involved in interacting. While $\mathrm{H}$-bonds and water bridges were dominant, hydrophobic interactions were also seen with Leu844, Phe795, Met1002, Tyr998 and Ala722. The total number of contacts fluctuated between 24 and 16 for the first half of the simulation, though after $60 \mathrm{~ns}$, the total number of contacts remained stable at an average of 20 . These results indicated that in all cases the mutated peptides had higher numbers of contacts with the receptor compared to the original peptide, which may help in enhanced binding. The overall ligand atom-protein residue interactions are shown in Supplementary Interaction Figure S12, which also confirms the interactions with the various residues of the receptor.

For the polyphenol-peptide conjugates, results for the protein-ligand contacts with EGFR are shown in Figure 14. For the RMA-Pep conjugate (Figure 14a), we observed a number of $\mathrm{H}$-bond and water-bridge interactions, in addition to few hydrophobic interactions with Phe723, Tyr998, Leu1001, Lys716, Val717, Leu799, Arg803 and Met1002.

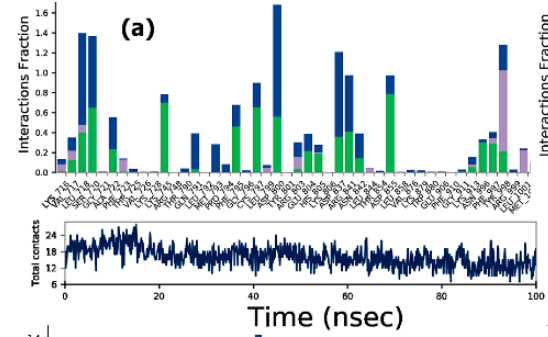

(c)


H-bonds $\square$ Hydrophobic $\square$ lonic $\square$ Water bridges (b)

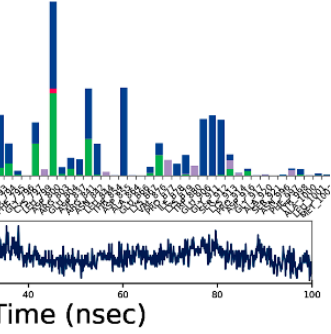

(d)

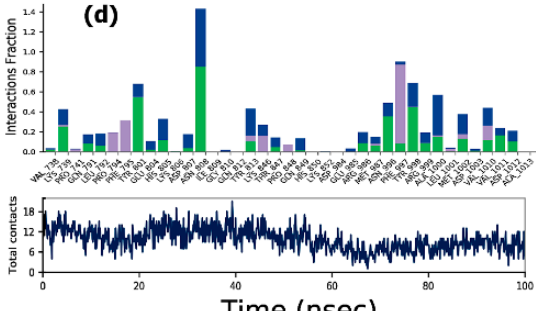

(f)

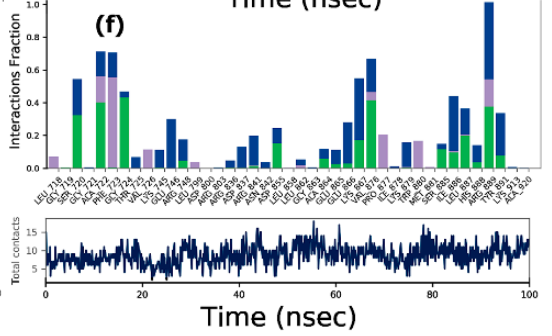

Figure 14. Protein ligand contacts of EGFR ligand complexes. (a) with RMAPep (top); total number of contacts with RMAPep (bottom); (b) with RMAMut1 (top); total number of contacts with RMAMut1 (bottom); (c) with GLAMut1 (top); total number of contacts with GLAMut1 (bottom); (d) with GLAMut2 (top); total number of contacts GLAMut2 (bottom); (e) with CGA-Mut4 (top); total number of contacts with CGA-Mut4 (bottom); (f) with CSAMut4 (top); total number of contacts with CSAMut4 (bottom). 
Once again, Asp800 showed a high interaction fraction (1.6), followed by Ser720 and Leu718. The total number of contacts throughout the simulation process was found to be in the range of 18-24 for the first $40 \mathrm{~ns}$, after which the number of contacts was found to be 16 on average. In comparison, RMAMut1 (Figure 14b) showed increased ionic interactions with a higher interaction fraction observed for Asp800 (2.5). Residues such as Pro877, Trp880, Lys913, Phe723, Ala722, Leu718, Ala743 and Leu799 were involved in hydrophobic interactions. The number of interactions throughout the simulation process was found to be 20 on average, with higher interactions observed in the first 40 ns of the simulation. GLAMut1 (Figure 14c) once again showed mostly H-bonds and water bridges with some hydrophobic interactions, while the total number of contacts throughout the simulation was found to be 14 on average, which is lower than other conjugates. This may be due to the way that the conjugate folds up in the receptor binding cleft. For GLAMut2, (Figure 14d) the highest interaction fraction was observed for Asn808. The prominent hydrophobic interactions were found to be with Phe997, Phe795, and Pro794. The overall average number of contacts was 10 throughout the simulation process. CGAMut4 (Figure 14e) showed a number of H-bond and water bridge interactions, though Ile715 showed a relatively high interaction fraction primarily for hydrophobic interaction along with Val716, Leu718, Val726 and Leu1001. The total number of receptor-ligand contacts was found to be 20 on average, with highest number of contacts (28) observed within the first 50 ns. CSAMut (Figure 14f) showed slightly different ligand contacts with the receptor. Interestingly, Asp800 was not the dominant interaction, in fact Asp800 showed the lowest interaction with CSAMut4. Instead, Arg889 had the highest interaction fraction. Several other interactions were observed which included mostly $\mathrm{H}$-bonds and water bridges and hydrophobic interactions with Phe 723, Leu718, Val726, Pro877, Trp880 and Arg889.

The total number of contacts throughout the simulation was lower, with an average of only 9 contacts over the simulation process. These results indicate that, among the conjugates, RMAMut 1 and CGAMut 4 were found to have the highest number of contacts throughout the simulation. The individual ligand atom-receptor contacts are also shown in Supplementary Information Figure S13, which further corroborates with the results seen from the histograms of the protein-ligand contacts.

\subsection{MM-GBSA Free Energy Studies}

The binding energy estimation using the molecular mechanics-generalized Born surface area (MMGBSA) method allows for identifying the ligands that bind efficiently [74] with receptors. The validity of the peptides and conjugates identified by docking and MD simulations was further explored by conducting MMGBSA binding free energy estimation calculations.

The major contributors, $\mathrm{H}$-bonding, lipophilic interactions, electrostatic interactions and van der Waals interaction energy calculations were also examined from the trajectories obtained during the simulation. The results obtained for the ligands with each of the receptors are shown in Table 5 and Figure 15. For ER- $\alpha$, the highest $\Delta \mathrm{G}$ bind was seen for the Mut4 peptide (YHWYGYTPQD) at $\Delta \mathrm{G}=-108.9 \mathrm{kcal} / \mathrm{mol}$. This value is significantly higher than that observed for the original peptide (YHWYGYTPQN). Thus, this single point mutation had a profound effect in enhancing $\Delta \mathrm{G}$ bind for estrogen receptor, likely due to enhanced electrostatic interactions. In addition, CGAMut4 and CSAMut4 also showed high $\Delta \mathrm{G}$ bind at $-80.9 \mathrm{kcal} / \mathrm{mol}$ and $-78.3 \mathrm{kcal} / \mathrm{mol}$, respectively, while the lowest $\Delta \mathrm{G}$ bind was observed for Mut 1 (FHWYGYTPQN), indicating that the single point mutation involving alteration of $\mathrm{Y}$ to $\mathrm{F}$ reduces $\Delta \mathrm{G}$ binding with the estrogen receptor. 
Table 5. Comparison of MMGBSA estimations.

\section{Estrogen-Alpha Receptor}

\begin{tabular}{|c|c|c|c|c|c|}
\hline Compound & $\begin{array}{l}\text { Avg. } \Delta G \text { Binding } \\
\text { Energy (kcal/mol) }\end{array}$ & $\begin{array}{l}\text { Avg Electrostatic } \\
\text { Energy (kcal/mol) }\end{array}$ & $\begin{array}{c}\text { Avg. H-Bond } \\
\text { Energy (kcal/mol) }\end{array}$ & $\begin{array}{c}\text { Avg. Lipophilic } \\
\text { Energy (kcal/mol) }\end{array}$ & $\begin{array}{l}\text { Avg. vdW Energy } \\
\text { (kcal/mol) }\end{array}$ \\
\hline Pep & -61.90 & -37.11 & -6.40 & -15.94 & -71.33 \\
\hline Mut1 & -37.97 & -24.44 & -2.52 & -11.21 & -36.98 \\
\hline Mut2 & -70.94 & -37.61 & -5.45 & -17.87 & -69.43 \\
\hline Mut3 & -70.62 & -39.03 & -5.48 & -18.91 & -61.08 \\
\hline Mut4 & -108.90 & -54.87 & -6.72 & -32.92 & -98.60 \\
\hline RMAPep & -59.98 & -38.10 & -6.70 & -15.77 & -70.52 \\
\hline GLAMut1 & -45.97 & -23.47 & -2.11 & -8.74 & -45.23 \\
\hline RMAMut1 & -41.62 & -30.82 & -3.29 & -12.04 & -42.22 \\
\hline GLAMut2 & -47.28 & -30.05 & -2.89 & -11.53 & -42.30 \\
\hline CGAMut4 & -80.92 & -61.98 & -6.34 & -18.01 & -72.00 \\
\hline CSAMut4 & -78.35 & -32.18 & -3.71 & -22.93 & -80.60 \\
\hline \multicolumn{6}{|c|}{ PPAR-Alpha Receptor } \\
\hline Pep & -69.14 & -18.34 & -4.73 & -20.88 & -64.80 \\
\hline Mut1 & -67.02 & -16.01 & -3.72 & -21.31 & -66.31 \\
\hline Mut2 & -67.78 & -5.03 & -3.37 & -20.63 & -64.29 \\
\hline Mut3 & -63.39 & -17.82 & -4.49 & -18.88 & -61.90 \\
\hline Mut4 & -63.02 & -16.45 & -3.66 & -19.56 & -62.26 \\
\hline RMAPep & -67.44 & -20.33 & -4.29 & -20.60 & -64.76 \\
\hline GLAMut1 & -71.47 & -33.03 & -4.99 & -21.30 & -66.18 \\
\hline RMAMut1 & -65.35 & -28.73 & -3.32 & -21.03 & -65.46 \\
\hline GLAMut2 & -67.35 & -43.97 & -4.39 & -22.28 & -69.34 \\
\hline CGAMut4 & -68.73 & -16.27 & -4.52 & -20.91 & -66.46 \\
\hline CSAMut4 & -40.20 & -9.80 & -1.95 & -12.81 & -39.70 \\
\hline \multicolumn{6}{|c|}{ EGFR Receptor } \\
\hline Pep & -174.54 & -48.74 & -8.32 & -30.00 & -114.43 \\
\hline Mut1 & -194.22 & -98.96 & -10.01 & -33.74 & -128.01 \\
\hline Mut2 & -173.50 & -54.84 & -8.41 & -29.92 & -113.84 \\
\hline Mut3 & -173.36 & -40.40 & -8.18 & -32.39 & -116.54 \\
\hline Mut4 & -188.66 & -69.22 & -8.52 & -33.58 & -124.70 \\
\hline RMAPep & -63.04 & -37.18 & -3.73 & -16.14 & -53.81 \\
\hline GLAMut1 & -175.16 & -59.21 & -8.33 & -30.74 & -118.13 \\
\hline RMAMut1 & -49.77 & -9.41 & -4.12 & -12.13 & -60.20 \\
\hline GLAMut2 & -173.75 & -39.93 & -7.70 & -32.65 & -117.88 \\
\hline CGAMut4 & -68.30 & -49.13 & -4.76 & -13.82 & 429.28 \\
\hline CSAMut4 & -46.60 & -23.03 & -1.87 & -15.77 & -44.96 \\
\hline
\end{tabular}



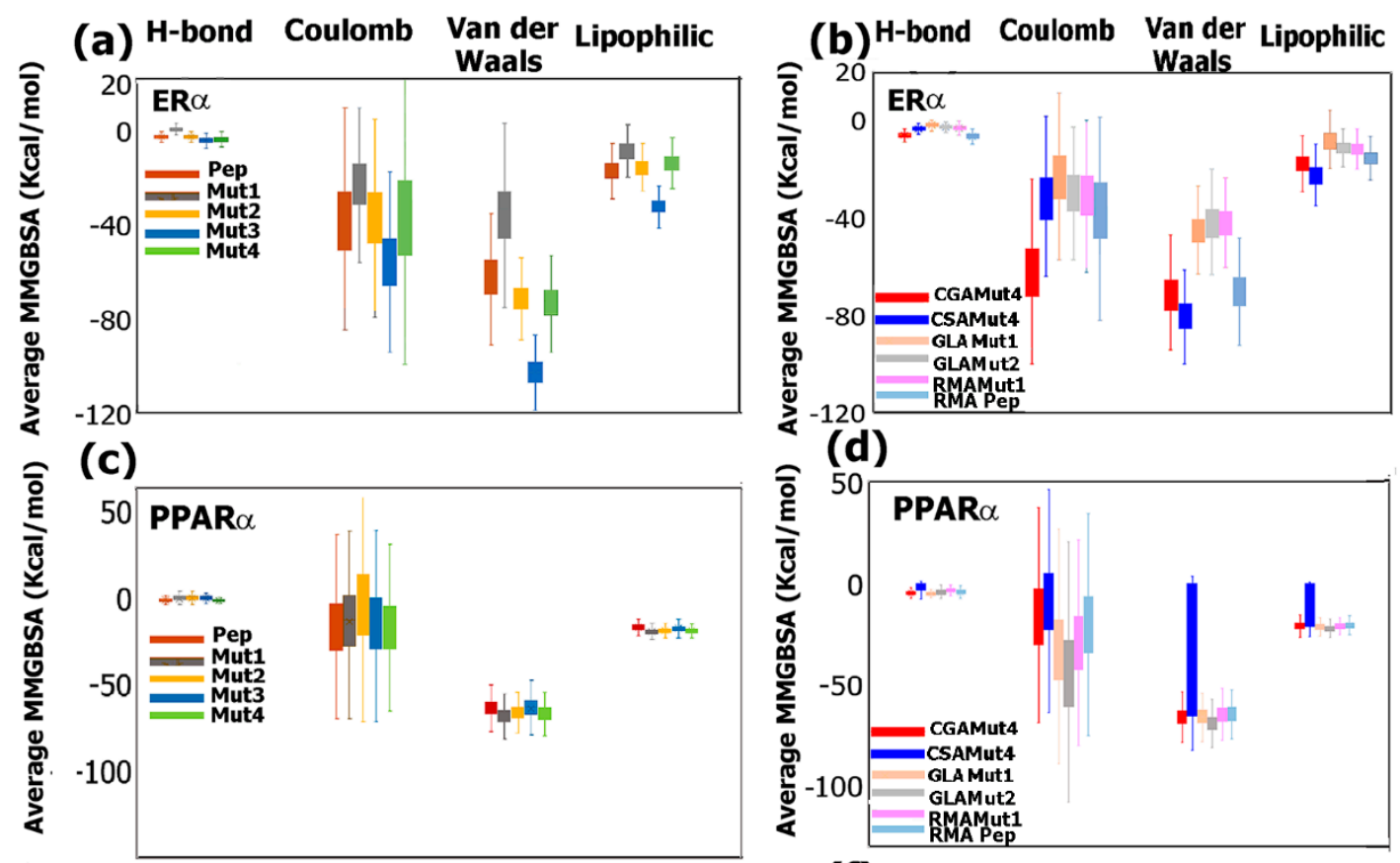

(e)
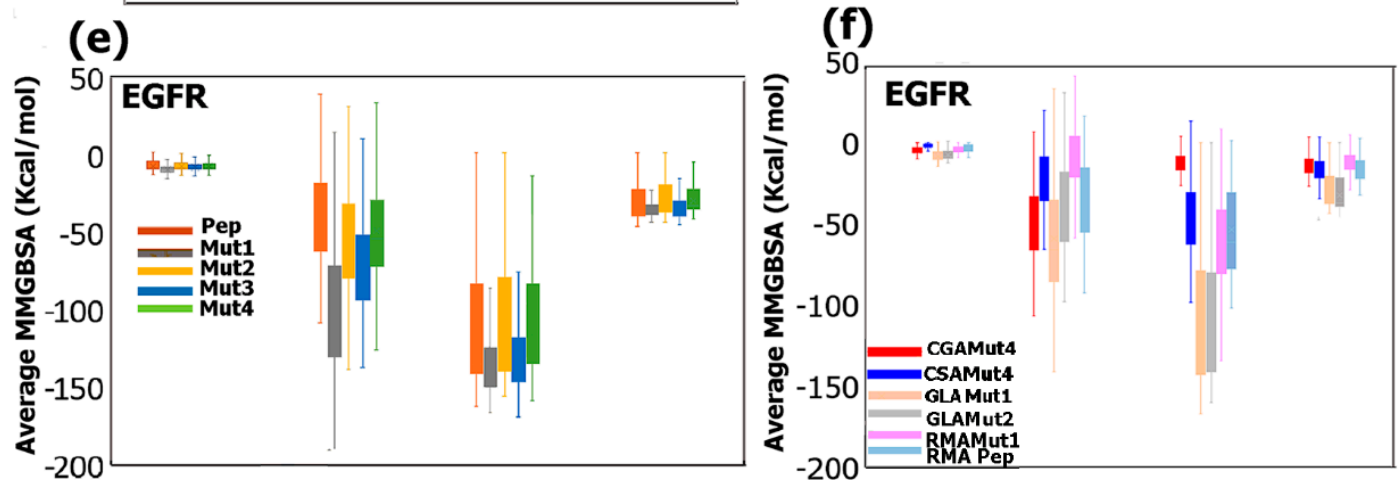

Figure 15. Comparison of $\Delta \mathrm{G}$ binding interactions based on MMGBSA calculations. H-bonding, coulomb interactions, van der Waals and lipophilic contributions are shown. (a,c,e) show interactions involving the peptides (Pep, and mutated peptides) while $(\mathbf{b}, \mathbf{d}, \mathbf{f})$ show the interactions with the polyphenol-conjugates of the peptides with $\mathrm{ER} \alpha, \operatorname{PPAR} \alpha$ and EGFR respectively.

In all cases, van der Waals interactions and electrostatic (coulomb) interactions were found to play a significant role in binding. For PPAR- $\alpha$, the $\Delta G$ bind was found to be in the range of -63.0 to $-71.5 \mathrm{kcal} / \mathrm{mol}$ for all of the peptides and the polyphenol conjugates, except for CSAMut4, which showed the lowest $\Delta \mathrm{G}$ bind at $-40.2 \mathrm{kcal} / \mathrm{mol}$. These results indicate that, overall, each of the ligands had a fairly moderate binding energy, and stability. Furthermore, conjugating with the polyphenols did not alter the binding significantly, except with CSA, where the binding affinity was lowered. Overall, these values obtained are similar to those obtained for $\Delta \mathrm{G}$ bind for kaempferol and resveratrol in a recent study that explored binding interactions of polyphenols with PPAR $\gamma$ using MMGBSA estimations [75]. It is well known that PPAR receptors- $\alpha$ and $\gamma$ have hydrophobic binding pockets, and the peptides and the polyphenol-peptide conjugates have hydrophobic regions. Additionally, the polyphenolic components increase $\mathrm{H}$-bonding interactions due to the phenolic $-\mathrm{OH}$ groups. The binding energies for the polyphenol-peptide conjugates GLAMut1, RMA-Pep, RMAMut1, and GLAMut2 were found to be higher for the PPAR- $\alpha$ compared to the ER $\alpha$ while CSAMut4 was lower despite the fact that it contains the diterpene moiety of the carnosic acid component. Since PPAR- $\alpha$ normally binds to fatty acid ligands, it is likely that the polyphenol groups may not interact strongly due to relatively lower hydrophobicity compared to long chain fatty acids. Nevertheless, most of the ligands showed binding 
energies that are close to other commercially available medicinal polyphenolic compounds, as mentioned above and may be probed as drug candidates.

Figure 15 further confirms that the most negative binding energies were found to be for van der Waals interactions, illustrating their role in the binding of ligands to each of the receptors with EGFR being showing the highest (most negative values) followed by ER- $\alpha$ and PPAR- $\alpha$. Coulombic forces also played a significant role, though their values were fairly similar for each of the receptor's ligands, with the exception of Mut1 which was more negative for EGFR. Furthermore, each of the ligands demonstrated by far the highest binding energies for EGFR compared to the ER- $\alpha$ and PPAR- $\alpha$. It was also demonstrated from docking studies as well as MD simulations, that these ligands attach firmly into the binding pocket of EGFR, encompassing a second sub pocket region as well $[75,76]$. The highest $\triangle \mathrm{G}$ bind was seen for Mut1 with EGFR at $-194.2 \mathrm{kcal} / \mathrm{mol}$, while the lowest value was observed for CSAMut 4 at $-146.6 \mathrm{kcal} / \mathrm{mol}$. These results corroborate with the MD studies as well as docking studies. The $\Delta \mathrm{G}$ bind values for RMAMut1 and RMA-original peptide were relatively lower at $-49.7 \mathrm{kcal} / \mathrm{mol}$ and at $-63.0 \mathrm{kcal} / \mathrm{mol}$ compared to most of the other ligands which showed $\Delta \mathrm{G}$ bind values $>-150 \mathrm{kcal} / \mathrm{mol}$. These high average binding energies indicate the stability of the ligands within the binding pockets of EGFR. Given that their values are close to or higher than the MMGBSA value obtained for the well-known EGFR inhibitor TAK-285 [77], we can propose that all the peptides as well as GLAMut1 and GLAMut2 may be probed further for biological studies for inhibition of EGFR.

\section{ADME Studies}

ADME studies to assess the pharmacokinetic properties of the peptides and selected conjugates are important in order to evaluate the potential of the designed compounds as drug candidates [78]. In particular, in silico ADME screening can provide information that aids in selecting the most promising compounds and minimize the possibility of drug rejection [79]. We therefore evaluated several critical parameters including partition coefficient $(\log P)$, Madin-Darby canine kidney cells (MDCK) permeability, ability to behave as Pgp inhibitor/substrate, CYP enzyme substrate/inhibitor capability and hERG blocker capability. MDCK has been widely developed as model for permeability and its apparent permeability coefficient, $\mathrm{P}_{\mathrm{app}}$, is widely considered to provide information about the uptake efficiency of compounds into cells [80]. Additionally, it is critical that the designed drugs do not show hERG blockage as hERG (human ether-a-go-go related gene) plays an important role in the regulation of the exchange of cardiac action potential and resting potential [81]

The results are listed in Table 6. Overall, our results indicated that the logP values increased for the conjugates in most cases compared to the individual peptides. All the peptides showed negative $\log \mathrm{P}$ values, of which Mut2 showed the highest lipophilicity at -0.407 and Pep showed the lowest lipophilicity at -1.698 . Among the conjugates, carnosate-Mut4, at a $\log P$ value of 2.145 , showed the highest lipophilicity followed by RMA-Mut1 at 0.452 and RMA-Pep at 0.046 . In general, LogP values of $0-3$ are considered to be optimal [81] and therefore the compounds RMA-Mut1; CSA-Mut4 and RMA-Pep would be considered to have optimal $\log \mathrm{P}$ values, though the other conjugates also have $\log \mathrm{P}$ values fairly close to zero. MDCK permeability was found to be the highest for Mut2, while all other candidates, with the exception of CGA-Mut4, RMA-Pep, CSA-Mut4 and Mut4, showed medium permeability ( $>2$ but $<20 \times 10^{-6} \mathrm{~cm} / \mathrm{s}$ ). CGA-Mut4, RMA-Pep, CSAMut4 and Mut4 showed permeability below $2 \times 10^{-6} \mathrm{~cm} / \mathrm{s}$ indicative of low permeability. The compounds did not form CYP substrates or inhibitors, though they did form PgP substrates in most cases, indicative of the fact that this property may affect drug efflux. None of the compounds caused hERG blockage, which is a desirable property in drugs. 
Table 6. ADME Studies.

\begin{tabular}{cccccc}
\hline Compound & $\begin{array}{c}\text { Log P } \\
\text { at pH 7.4 }\end{array}$ & $\begin{array}{c}\text { MDCK Permeability } \\
\mathbf{( c m} / \mathbf{s})\end{array}$ & $\begin{array}{c}\text { hERG } \\
\text { Blocker }\end{array}$ & $\begin{array}{c}\text { Pgp Inhibitor/ } \\
\text { Substrate }\end{array}$ & $\begin{array}{c}\text { CYP1A2 Substrate } \\
\text { /or Inhibitor }\end{array}$ \\
\hline Pep (Y-H-W-Y-G-Y-T-P-Q-N) & -1.698 & $2.5 \times 10^{-6}$ & $\mathrm{No}$ & $\mathrm{No} / \mathrm{No}$ & $\mathrm{No} / \mathrm{No}$ \\
\hline Mut 1 (F-H-W-Y-G-Y-T-P-Q-N) & -0.962 & $2.9 \times 10^{-6}$ & $\mathrm{No}$ & $\mathrm{No} / \mathrm{Yes}$ & $\mathrm{No} / \mathrm{No}$ \\
\hline Mut 2 (Y-I-W-Y-G-Y-T-P-Q-N) & -0.407 & $3.2 \times 10^{-6}$ & $\mathrm{No}$ & $\mathrm{No} / \mathrm{Yes}$ & $\mathrm{No} / \mathrm{No}$ \\
\hline Mut 3 (Y-H-W-Y-G-Y-T-H-Q-N) & -2.112 & $2 \times 10^{-6}$ & $\mathrm{No}$ & $\mathrm{No} / \mathrm{Yes}$ & $\mathrm{No} / \mathrm{No}$ \\
\hline Mut 4 (Y-H-W-Y-G-Y-T-P-Q-D) & -1.487 & $1.9 \times 10^{-6}$ & $\mathrm{No}$ & $\mathrm{No} / \mathrm{Yes}$ & $\mathrm{No} / \mathrm{No}$ \\
\hline Rosmarinate-Peptide & 0.046 & $1.9 \times 10^{-6}$ & $\mathrm{No}$ & $\mathrm{No} / \mathrm{Yes}$ & $\mathrm{No} / \mathrm{No}$ \\
\hline Gallate-Mut 1 & -0.565 & $2.2 \times 10^{-6}$ & $\mathrm{No}$ & $\mathrm{No} / \mathrm{Yes}$ & $\mathrm{No} / \mathrm{Yes}$ \\
\hline Rosmarinate-Mutation 1 & 0.452 & $2.1 \times 10^{-6}$ & $\mathrm{No}$ & $\mathrm{No} / \mathrm{Yes}$ & $\mathrm{No} / \mathrm{No}$ \\
\hline Gallate-Mut 2 & -0.070 & $2.1 \times 10^{-6}$ & $\mathrm{No}$ & $\mathrm{No}$ & $\mathrm{No} / \mathrm{Yes}$ \\
\hline Chlorogenate-Mut 4 & -0.089 & $1.4 \times 10^{-6}$ & $\mathrm{No}$ & $\mathrm{No} / \mathrm{Yes}$ & $\mathrm{No} / \mathrm{No}$ \\
\hline Carnosate-Mut 4 & 2.145 & $1.8 \times 10^{-6}$ & $\mathrm{No}$ & $\mathrm{No}$ \\
\hline
\end{tabular}

\section{Conclusions}

In this study, we have shown that the peptide sequence YHWYGYTPQN, previously known to bind to EGFR, is also capable of binding to ER $\alpha$ and PPAR $\alpha$, making it suitable for targeting multiple over-expressed receptors in tumor cells. We have further demonstrated that four point mutations of this peptide sequence and conjugation of both this sequence and mutated sequences to polyphenols, such as gallic acid and rosmarinic acid, can further enhance binding interactions of with the three receptors. In the case of the EGFR receptor, most of the conjugates appeared to bind to the kinase domain binding pocket region, while, in the case of $E R \alpha$, binding occurred at the alpha-helical sandwich region of the binding pocket. Of the twenty novel peptide-polyphenol conjugates studied, those found to be most promising for ER $\alpha$, PPAR $\alpha$, and EGFR targeting based on molecular docking and molecular dynamics results were rosmarinate-YHWYGYTPQN, gallate- YIWYGYTPQN and gallateFHWYGYTPQN. MMGBSA studies revealed that the lowest binding energies (therefore, the highest binding interactions) were found to be with Pep (YHWYGYTPQN) and Mut1 (FHWYGYTPQN), GLAMut1 and GLAMut2 for the EGFR receptor, while GLAMut1 also showed low binding energy with PPAR- $\alpha$ receptor. Mut4 and CGAMut 4 showed the lowest binding energies with ER- $\alpha$. Thus, each of these peptides and conjugates can also be fine-tuned to bind to the specific receptors. ADME studies revealed that the lipophilicity was increased upon conjugation of the peptides with the polyphenols. Most of the designed compounds showed medium permeability for MDCK cells. Though all of the conjugates formed Pgp substrates, none formed CYPA1/A2 substrates or inhibitors. Overall, the polyphenol peptide conjugates (particularly GLAMut2) and (RMAPep) designed here can be potentially explored for further synthesis and exploration as drug candidates for targeting breast cancer cells.

Supplementary Materials: The following supporting information can be downloaded at: https: / /www.mdpi.com/article/10.3390/app12010515/s1, Figures S1-S13: RMSDs, Trajectory images for MD simulations of Pep; the four mutated peptides as well as the selected polyphenol-peptide conjugates at $0,25,50,75$ and 100 ns showing interactions of the peptides with each of the receptors; as well as schematic of ligand-atom interactions with each of the receptors. Table S1-Binding energies obtained from FireDock analysis; Tables S2-S7 shows PLIP results for each of the peptides and the polyphenol-peptide conjugates. 
Author Contributions: Conceptualization: I.A.B.; methodology and data acquisition I.A.B., S.M.M., L.R.H., C.G.L. and R.E.D.; validation, S.M.M., L.R.H., C.G.L. and I.A.B.; formal analysis, L.R.H. and I.A.B.; writing—original draft preparation, L.R.H., C.G.L. and I.A.B.—review and editing, I.A.B. and L.R.H.; supervision, I.A.B.; project administration, I.A.B.; funding acquisition, L.R.H. and C.G.L. All authors have read and agreed to the published version of the manuscript.

Funding: This research was funded by Fordham University Research Grants.

Institutional Review Board Statement: Not applicable.

Informed Consent Statement: Not applicable.

Data Availability Statement: The data presented in this study are available in Supplementary Information.

Acknowledgments: The authors thank U. Thaduri at Fordham IT for technical support.

Conflicts of Interest: The authors declare no conflict of interest.

\section{References}

1. Xiao, Y.F.; Jie, M.M.; Li, B.S.; Hu, C.J.; Xie, R.; Tang, B.; Yang, S.M. Peptide-Based Treatment: A Promising Cancer Therapy. J. Immunol. Res. 2015, 2015, e761820. [CrossRef] [PubMed]

2. Le Joncour, V.; Laakkonen, P. Seek \& Destroy, use of targeting peptides for cancer detection and drug delivery. Bioorg. Med. Chem. 2018, 26, 2797-2806.

3. Scodeller, P.; Asciutto, E.K. Targeting Tumors Using Peptides. Molecules 2020, 25, 808. [CrossRef] [PubMed]

4. Yuan, X.; Xu, Y. Recent trends and applications of molecular modeling in GPCR-ligand recognition and structure based design. Int. J. Mol. Sci. 2018, 19, 2105. [CrossRef]

5. Kostrzewa, T.; Sahu, K.K.; Gorska-Ponikowska, M.; Tuszynski, J.A.; Kuban-Jankowska, A. Synthesis of small peptide compounds, molecular docking, and inhibitory activity evaluation against phosphatases PTP1B and SHP2. Drug Des. Devel. Ther. 2018, 12, 4139-4147. [CrossRef] [PubMed]

6. $\quad$ Feiner, R.C.; Kemker, I.; Krutzke, L.; Allmendinger, E.; Mandell, D.J.; Sewald, N.; Kochanek, S.; Müller, K.M. EGFR-Binding Peptides: From Computational Design towards Tumor-Targeting of Adeno-Associated Virus Capsids. Int. J. Mol. Sci. 2020, 21, 9535. [CrossRef] [PubMed]

7. Aranda, F.; Vacchelli, E.; Eggermont, A.; Galon, J.; Sautès-Fridman, C.; Tartour, E.; Zitvogel, L.; Kroemer, G.; Galluzzi, L. Trial Watch: Peptide vaccines in cancer therapy. OncoImmunology 2013, 2, e26621. [CrossRef]

8. Hoppenz, P.; Els-Heindl, S.; Beck-Sickinger, A.G. Peptide-Drug Conjugates and Their Targets in Advanced Cancer Therapies. Front. Chem. 2020, 8, 571. [CrossRef]

9. Jaracz, S.; Chen, J.; Kuznetsova, L.V.; Ojima, I. Recent Advances in Tumor-Targeting Anticancer Drug Conjugates. Bioorg. Med. Chem. 2005, 13, 5043-5054. [CrossRef]

10. Cooper, B.M.; Iegre, J.; Donovan, D.H.O.; Halvarsson, M.Ö.; Spring, D.R. Peptides as a platform for targeted therapeutics for cancer: Peptide-drug conjugates (PDCs). Chem. Soc. Rev. 2021, 50, 1480-1494. [CrossRef]

11. He, R.; Finan, B.; Mayer, J.P.; DiMarchi, R.D. Peptide Conjugates with Small Molecules Designed to Enhance Efficacy and Safety. Molecules 2019, 24, 1855. [CrossRef]

12. Song, Q.; Chuan, X.; Chen, B.; He, B.; Zhang, H.; Dai, W.; Wang, X.; Zhang, Q. A Smart Tumor Targeting Peptide-Drug Conjugate, PHLIP-SS-DOX: Synthesis and Cellular Uptake on MCF-7 and MCF-7/Adr Cells. Drug Deliv. 2016, 23, 1734-1746. [CrossRef]

13. Gilad, Y.; Noy, E.; Senderowitz, H.; Albeck, A.; Firer, M.A.; Gellerman, G. Synthesis, biological studies and molecular dynamics of new anticancer RGD-based peptide conjugates for targeted drug delivery. Bioorg. Med. Chem. 2016, 24, 294-303. [CrossRef] [PubMed]

14. Azqueta, A.; Collins, A. Polyphenols and DNA Damage: A Mixed Blessing. Nutrients 2016, 8, 785. [CrossRef]

15. Shaikh, A.A.; Braakhuis, A.J.; Bishop, K.S. The Mediterranean Diet and Breast Cancer: A Personalised Approach. Healthcare 2019, 7, 104. [CrossRef] [PubMed]

16. Bhosale, P.B.; Ha, S.E.; Vetrivel, P.; Kim, H.H.; Kim, S.M.; Kim, G.S. Functions of polyphenols and its anticancer properties in biomedical research: A narrative review. Transl. Cancer Res. 2020, 9, 7619-7631. [CrossRef]

17. Yesil-Celiktas, O.; Sevimli, C.; Bedir, E.; Vardar-Sukan, F. Inhibitory Effects of Rosemary Extracts, Carnosic Acid and Rosmarinic Acid on the Growth of Various Human Cancer Cell Line. Plant Foods Hum. Nutr. 2010, 65, 158-163. [CrossRef]

18. Meng, S.; Cao, J.; Feng, Q.; Peng, J.; Hu, Y. Roles of Chlorogenic Acid on Regulating Glucose and Lipids Metabolism: A Review. Evid. Based Complement Alternat. Med. 2013, 2013, 801457. [CrossRef]

19. Faried, A.; Kurnia, D.; Faried, L.; Usman, N.; Miyazaki, T.; Kato, H.; Kuwano, H. Anticancer effects of gallic acid isolated from Indonesian herbal medicine, Phaleria macrocarpa (Scheff.) Boerl, on human cancer cell lines. Int. J. Oncol. 2007, 30, 605-613. [CrossRef]

20. Fantini, M.; Benvenuto, M.; Masuelli, L.; Frajese, G.V.; Tresoldi, I.; Modesti, A.; Bei, R. In Vitro and in Vivo Antitumoral Effects of Combinations of Polyphenols, or Polyphenols and Anticancer Drugs: Perspectives on Cancer Treatment. Int. J. Mol. Sci. 2015, 16, 9236-9282. [CrossRef] 
21. Chen, H.; Yao, K.; Nadas, J.; Bode, A.M.; Malakhova, M.; Oi, N.; Li, H.; Lubet, R.A.; Dong, Z. Prediction of Molecular Targets of Cancer Preventing Flavonoid Compounds Using Computational Methods. PLoS ONE 2012, 7, e38261. [CrossRef]

22. Chen, H.; Yao, K.; Chang, X.; Shim, J.; Kim, H.; Malakhova, M.; Kim, D.; Bode, A.M.; Dong, Z. Computational and Biochemical Discovery of RSK2 as a Novel Target for Epigallocatechin Gallate (EGCG). PLoS ONE 2015, 10, e0130049. [CrossRef] [PubMed]

23. Aminpour, M.; Montemagno, C.; Tuszynski, J.A. An Overview of Molecular Modeling for Drug Discovery with Specific Illustrative Examples of Applications. Molecules 2019, 24, 1693. [CrossRef] [PubMed]

24. Salmaso, V.; Moro, S. Bridging Molecular Docking to Molecular Dynamics in Exploring Ligand-Protein Recognition Process: An Overview. Front. Pharmacol. 2018, 9, 923. [CrossRef] [PubMed]

25. Ghosh, R.; Chakraborty, A.; Biswas, A.; Chowdhuri, S. Identification of polyphenols from Broussonetia papyrifera as SARS CoV-2 main protease inhibitors using in silico docking and molecular dynamics simulation approaches. J. Biomol. Struct. Dynamics. 2020, 39, 6747-6760. [CrossRef] [PubMed]

26. Manivannan, A.; Soundararajan, P.; Park, Y.G.; Sakkiah, S.; Jeong, B.R. Binding Mode Investigation of Polyphenols from Scrophularia Targeting Human Aldose Reductase Using Molecular Docking and Molecular Dynamics Simulations. J. Chem. 2015, 2015, e434256. [CrossRef]

27. Tan, Y.; Wang, M.; Yang, K.; Chi, T.; Liao, Z.; Wei, P. PPAR- $\alpha$ Modulators as Current and Potential Cancer Treatments. Front. Oncol. 2021, 11, e599995. [CrossRef]

28. Costa, R.; Shah, A.N.; Santa-Maria, C.A.; Cruz, M.R.; Mahalingam, D.; Carneiro, B.A.; Chae, Y.K.; Cristofanilli, M.; Gradishar, W.J.; Giles, F.J. Targeting epidermal growth factor receptor in triple negative breast cancer: New discoveries and practical insights for drug development. Cancer Treat. Rev. 2017, 53, 111-119. [CrossRef] [PubMed]

29. Hossein-Nejad-Ariani, H.; Althagafi, E.; Kaur, K. Small Peptide Ligands for Targeting EGFR in Triple Negative Breast Cancer Cells. Sci. Rep. 2019, 9, 2723. [CrossRef]

30. Skandalis, S.S.; Afratis, N.; Smirlaki, G.; Nikitovic, D.; Theocharis, A.D.; Tzanakakis, G.N.; Karamanos, N.K. Cross-talk between estradiol receptor and EGFR/IGF-IR signaling pathways in estrogen-responsive breast cancers: Focus on the role and impact of proteoglycans. Matrix Biol. 2014, 35, 182-193. [CrossRef]

31. Jeong, J.; Kim, H.; Choi, J. In Silico Molecular Docking and In Vivo Validation with Caenorhabditis elegans to Discover Molecular Initiating Events in Adverse Outcome Pathway Framework: Case Study on Endocrine-Disrupting Chemicals with Estrogen and Androgen Receptors. Int. J. Mol. Sci. 2019, 20, 1209. [CrossRef]

32. Vijaykumar, S.; Ptv, L. ACPP: A webserver for prediction and design of anticancer peptides. Int. J. Pept. Res. Ther. 2015, 21, 99-106. [CrossRef]

33. Tyagi, A.; Kapoor, P.; Kumar, R.; Chaudhary, K.; Gautam, A. Raghava GPS. In Silico Models for Designing and Discovering Novel Anticancer Peptides. Sci. Rep. 2013, 3, 2984. [CrossRef] [PubMed]

34. The PyMOL Molecular Graphics System, Version 2.0; Schrödinger LLC: New York, NY, USA, 2020.

35. Yang, J.; Yan, R.; Roy, A.; Xu, D.; Poisson, J.; Zhang, Y. The I-TASSER Suite: Protein structure and function prediction. Nat. Methods 2015, 12, 7-8. [CrossRef]

36. Zhang, Y. I-TASSER server for protein 3D structure prediction. BMC Bioinform. 2008, 9, 40. [CrossRef] [PubMed]

37. Roy, A.; Kucukural, A.; Zhang, Y. I-TASSER: A unified platform for automated protein structure and function prediction. Nat. Protoc. 2010, 5, 725-738. [CrossRef] [PubMed]

38. Yang, J.; Roy, A.; Zhang, Y. Protein-ligand binding site recognition using complementary binding-specific substructure comparison and sequence profile alignment. Bioinformatics 2013, 29, 2588-2595. [CrossRef] [PubMed]

39. Yu, J.; Zhou, Y.; Tanaka, I.; Yao, M. Roll: A new algorithm for the detection of protein pockets and cavities with a rolling probe sphere. Bioinformatics 2010, 26, 46-52. [CrossRef]

40. Cipolletti, M.; Fernandez, V.S.; Montalesi, E.; Marino, M.; Fiocchetti, M. Beyond the Antioxidant Activity of Dietary Polyphenols in Cancer: The Modulation of Estrogen Receptors (ERs) Signaling. Int. J. Mol. Sci. 2018, 19, 2624. [CrossRef] [PubMed]

41. Sierra, M.L.; Beneton, V.; Boullay, A.B.; Boyer, T.; Brewster, A.G.; Donche, F.; Forest, M.C.; Fouchet, M.H.; Gellibert, F.J.; Grillot, D.A.; et al. Substituted 2-[(4-Aminomethyl)Phenoxy]-2-Methylpropionic Acid PPAR $\alpha$ Agonists. 1. Discovery of a Novel Series of Potent HDLc Raising Agent. J. Med. Chem. 2007, 50, 685-695. [CrossRef]

42. Xu, G.; Abad, M.C.; Connolly, P.J.; Neeper, M.P.; Struble, G.T.; Springer, B.A.; Emanuel, S.L.; Pandey, N.; Gruninger, R.H.; Adams, M.; et al. 4-Amino-6-arylamino-pyrimidine-5-carbaldehyde hydrazones as potent ErbB-2/EGFR dual kinase inhibitors. Bioorg. Med. Chem. Lett. 2008, 18, 4615-4619. [CrossRef]

43. Trott, O.; Olson, A.J. AutoDock Vina: Improving the Speed and Accuracy of Docking with a New Scoring Function, Efficient Optimization and Multithreading. J. Comput. Chem. 2010, 31, 455-461. [CrossRef]

44. Schneidman, D.; Nussinov, R.; Wolfson, H.J. Efficient Unbound Docking of Rigid Molecules. In Proceedings of the Algorithms in Bioinformatics, Second International Workshop, WABI, Rome, Italy, 17-21 September 2002; Volume 2542, pp. 185-200.

45. Mashiach, E.; Schneidman-Duhovny, D.; Andrusier, N.; Nussinov, R.; Wolfson, H.J. FireDock: A Web Server for Fast Interaction Refinement in Molecular Docking. Nucleic Acids Res. 2008, 36, W229-W232. [CrossRef] [PubMed]

46. Adasme, M.F.; Linnemann, K.L.; Bolz, S.N.; Kaiser, F.; Salentin, S.; Haupt, V.J.; Schroeder, M. PLIP 2021: Expanding the scope of the protein-ligand interaction profiler to DNA and RNA. Nucleic Acids Res. 2021, 49, W530-W534. [CrossRef]

47. Pérez-Benito, L.; Keränen, H.; van Vlijmen, H.; Tresadern, G. Predicting Binding Free Energies of PDE2 Inhibitors. The Difficulties of Protein Conformation. Sci. Rep. 2018, 8, 4883. [CrossRef] [PubMed] 
48. Venugopal, P.P.; Das, B.K.; Soorya, E.; Chakraborty, D. Effect of hydrophobic and hydrogen bonding interactions on the potency of ss-alanine analogs of G-protein coupled glucagon receptor inhibitors. Proteins 2020, 88, 327-344. [CrossRef] [PubMed]

49. Genheden, S.; Ryde, U. The MM/PBSA and MM/GBSA methods to estimate ligand-binding affinities. Expert Opin. Drug Discov. 2015, 10, 440-461. [CrossRef]

50. Du, J.; Sun, H.; Xi, L.; Li, J.; Yang, Y.; Liu, H.; Yao, X. Molecular modeling study of checkpoint kinase 1 inhibitors by multiple docking strategies and prime/MM GBSA calculation. J. Comp. Chem. 2011, 32, 2800-2809. [CrossRef]

51. Xiong, G.; Wu, Z.; Yi, J.; Fu, L.; Yang, Z.; Hsieh, C.; Yin, M.; Zeng, X.; Wu, C.; Chen, X.; et al. ADMETlab 2.0: An integrated online platform for accurate and comprehensive predictions of ADMET properties. Nucleic Acids Res. 2021, 49, W5-W14. [CrossRef]

52. Puranik, N.V.; Srivastava, P.; Bhatt, G.; Mary, D.J.S.J.; Limaye, A.M.; Sivaraman, J. Determination and Analysis of Agonist and Antagonist Potential of Naturally Occurring Flavonoids for Estrogen Receptor (ER $\alpha$ ) by Various Parameters and Molecular Modelling Approach. Sci. Rep. 2019, 9, 7450. [CrossRef]

53. Ma, Y.; Wang, S.; Xu, W.; Wang, R.; Chou, K. Design novel dual agonists for treating type-2 diabetes by targeting peroxisome proliferator-activated receptors with core hopping approach. PLoS ONE 2012, 7, e38546. [CrossRef]

54. Chen, G.; Seukep, A.J.; Guo, M. Recent advances in molecular docking for the research and discovery of potential marine drugs. Mar. Drugs. 2020, 18, 545. [CrossRef]

55. Grahl, M.V.C.; Alcará, A.M.; Perin, A.P.A.; Moro, C.F.; Pinto, É.S.M.; Feltes, B.C.; Ghilardi, I.M.; Rodrigues, F.V.F.; Dorn, M.; da Costa, J.C.; et al. Evaluation of drug repositioning by molecular docking of pharmaceutical resources available in the brazilian healthcare systems against SARS-CoV2. Inform. Med. Unlocked 2021, 23, 100539. [CrossRef]

56. Bianco, G.; Forli, S.; Goodsell, D.S.; Olson, A.J. Covalent docking using autodock: Two-point attractor and flexible side chain methods. Protein Sci. 2016, 25, 295-301. [CrossRef]

57. Yudt, M.R.; Vorojeikina, D.; Zhong, L.; Skafar, D.F.; Sasson, S.; Gasiewicz, T.A.; Notides, A.C. Function of estrogen receptor tyrosine 537 in hormone binding, DNA binding and transactivation. Biochemistry 1999, 38, 14146-14156. [CrossRef] [PubMed]

58. Grande, F.; Rizzuti, B.; Occhiuzzi, M.A.; Ioele, G.; Casacchia, T.; Gelmini, F.; Guzzi, R.; Garofalo, A.; Statti, G. Identification by Molecular Docking of Homoisoflavones from Leopoldia comosa as Ligands of Estrogen Receptors. Molecules 2018, $23,894$. [CrossRef]

59. Lambrinidis, G.; Halabalaki, M.; Katsanou, E.S.; Skaltsounis, A.-L.; Alexis, M.N.; Mikros, E. The estrogen receptor and polyphenos: Molecular simulation studies of their interactions, a review. Environ. Chem. Lett. 2006, 4, 159-174. [CrossRef]

60. Youssef, J.; Badr, M. Peroxisome proliferator-activated receptors and cancer: Challenges and opportunities. Br. J. Pharmacol. 2011, 164, 68-82. [CrossRef]

61. Lalloyer, F.; Staels, B. Fibrates, glitazones, and peroxisome proliferator-activated receptors. Arterioscler. Thromb. Vasc. Biol. 2010, 30, 894-899. [CrossRef] [PubMed]

62. Varga, T.; Czimmerer, Z.; Nagy, L. PPARs are a unique set of fatty acid regulated transcription factors controlling both lipid metabolism and inflammation. Biochim. Biophys. Acta 2011, 1812, 1007-1022. [CrossRef]

63. Cronet, P.; Petersen, J.F.; Folmer, R.; Blomberg, N.; Sjöblom, K.; Karlsson, U.; Lindstedt, E.L.; Bamberg, K. Structure of the PPAR $\alpha$ and $-\gamma$ ligand binding domain in complex with AZ 242; ligand selectivity and agonist activation in the PPAR family. Structure 2001, 9, 699-706. [CrossRef]

64. Ugwu, D.I.; Okoro, U.C.; Mishra, N.K.; Okafor, S.N. Novel Phenoxazinones as Potent Agonist of PPAR- $\alpha$ : Design, Synthesis, Molecular Docking and in Vivo Studies. Lipids Health Dis. 2018, 17, 120. [CrossRef]

65. Jura, N.; Zhang, X.; Endres, N.F.; Seeliger, M.A.; Schindler, T.; Kuriyan, J. Catalytic control in the EGF receptor and its connection to general kinase regulatory mechanisms. Mol. Cell 2011, 42, 9-22. [CrossRef]

66. Kumar, R.; Zakharov, M.N.; Khan, S.H.; Miki, R.; Jang, H.; Toraldo, G.; Singh, R.; Bhasin, S.; Jasuja, R. The Dynamic Structure of the Estrogen Receptor. J. Amino Acids 2011, 2011, 812540. [CrossRef]

67. Brzozowski, A.M.; Pike, A.C.W.; Dauter, Z.; Hubbard, R.E.; Bonn, T.; Engström, O.; Öhman, L.; Greene, G.L.; Gustafsson, J.; Carlquist, M. Molecular basis of agonism and antagonism in the oestrogen receptor. Nature 1997, 389, 753-758. [CrossRef]

68. Farooq, A. Structural and functional diversity of estrogen receptor ligands. Curr. Top. Med. Chem. 2015, 15, 1372-1384. [CrossRef] [PubMed]

69. Xu, H.E.; Lambert, M.H.; Montana, V.G.; Plunket, K.D.; Moore, L.B.; Collins, J.L.; Oplinger, J.A.; Kliewer, S.A.; Gampe, R.T.; McKee, D.D.; et al. Structural determinants of ligand binding selectivity between the peroxisome proliferator-activated receptors. Proc. Natl. Acad. Sci. USA 2001, 98, 13919-13924. [CrossRef]

70. Martin-Fernandez, M.L.; Clarke, D.T.; Roberts, S.K.; Zanetti-Domingues, L.C.; Gervasio, F.L. Structure and Dynamics of the EGF Receptor as Revealed by Experiments and Simulations and its Relevance to Non-Small Cell Lung Cancer. Cells $2019,8,316$. [CrossRef] [PubMed]

71. Hasenahuer, M.A.; Barletta, G.P.; Fernandez-Alberti, S.; Parisi, G.; Fornasari, M.S. Pockets as structural descriptors of EGFR kinase conformations. PLoS ONE 2017, 12, e0189147. [CrossRef] [PubMed]

72. Durai, P.; Govindaraj, R.G.; Choi, S. Structure and dynamic behavior of Toll-like receptor 2 subfamily triggered by malarial glycosylphosphatidylinostiols of Plasmodium falciparum. FEBS J. 2013, 280, 6196-6212. [CrossRef]

73. Kambia, N.; Farce, A.; Belarbi, K.; Gressier, B.; Luyckx, M.; Chavatte, P.; Dine, T. Dokcking Study: PPARs interaction with selected alternative plasticizers to di(2-ethylhexyl) phthalate. J. Enzyme Inhib. Med. Chem. 2016, 13, 448-455. 
74. Ahinko, M.; Niinivehmas, S.; Jokinen, E.; Pentikäinen, O.T. Suitability of MMGBSA for the selection of correct ligand binding modes from docking results. Chem. Biol. Drug Des. 2019, 93, 522-538. [CrossRef]

75. Lokhande, K.B.; Ballav, S.; Yadav, R.S.; Venkateswara Swamy, K.; Basu, S. Probing intermolecular interactions and binding stability of kaempferol, quercetin and resveratrol derivatives with PPAR- $\gamma$ : Docking, molecular dynamics and MM/GBSA approach to reveal potent PPAR- $\gamma$ agonist against cancer. J. Biomol. Struc. Dyn. 2020, 1-11. [CrossRef]

76. Sharma, V.K.; Nandekar, P.P.; Sangamwar, A.; Pérez-Sánchez, H.; Agarwal, S.M. Structure guided design and binding analysis of EGFR inhibiting analogues of erlotinib and AEE788 using ensemble docking, molecular dynamics and MMGBSA. RSC Adv. 2016, 6, 65725-65735. [CrossRef]

77. Al-Anazi, M.; Al-Najjar, B.O.; Khairuddean, M. Structure-based drug design studies toward the discovery of novel chalcone derivatives as potential epidermal growth factor receptor (EGFR) inhibitors. Molecules 2018, 23, 3203. [CrossRef] [PubMed]

78. Morcoss, M.M.; Abdelhafez, E.S.M.N.; Ibrahem, R.A.; Abdel-Rahman, H.M.; Abdel-Aziz, M.; Abou El-Ella, D.A. Design, synthesis, mechanistic studies and in silico ADME predictions of benzimidazole derivatives as novel antifungal agents. Bioorg. Chem. 2020, 101, 103956. [CrossRef] [PubMed]

79. Cheng, F.; Li, W.; Zhou, Y.; Shen, J.; Wu, Z.; Liu, G.; Lee, P.W.; Tang, Y. ADME-SAR: A comprehensive source and free tool for assessment of chemical ADMET properties. J. Chem. Inf. Model. 2012, 52, 3099-3105. [CrossRef] [PubMed]

80. Patel, R.D.; Kumar, S.P.; Pandya, H.A.; Solanki, H.A. MDCKpred: A web-tool to calculate MDCK permeability coefficient of small molecule using membrane-interaction chemical features. Toxicol. Mech. Methods 2018, 28, 685-698. [CrossRef] [PubMed]

81. Lu, D.; Chambers, P.; Wipf, P.; Xie, X.Q.; Englert, D.; Weber, S. Lipophilicity screening of novel drug-like compounds and comparison of cLogP. J. Chromatogr. A 2012, 1258, 161-167. [CrossRef] [PubMed] 